\title{
A Numerical Study of the Forces on Two Tandem Cylinders Exerted by Internal Solitary Waves
}

\author{
Yin Wang, ${ }^{1,2,3}$ Lingling Wang, ${ }^{1,2}$ Hai Zhu, ${ }^{1,2}$ Hongwu Tang, ${ }^{1,2}$ and Gang Wei ${ }^{4}$ \\ ${ }^{1}$ State Key Laboratory of Hydrology-Water Resources and Hydraulic Engineering, Hohai University, Nanjing 210098, China \\ ${ }^{2}$ College of Water Conservancy and Hydropower Engineering, Hohai University, Nanjing 210098, China \\ ${ }^{3}$ College of Water Conservancy and Ecological Engineering, Nanchang Institute of Technology, Nanchang 330099, China \\ ${ }^{4}$ College of Meteorology and Oceanography, PLA University of Science and Technology, Nanjing 211101, China
}

Correspondence should be addressed to Lingling Wang; wanglingling@hhu.edu.cn

Received 20 January 2016; Revised 9 May 2016; Accepted 22 May 2016

Academic Editor: Maurizio Brocchini

Copyright (C) 2016 Yin Wang et al. This is an open access article distributed under the Creative Commons Attribution License, which permits unrestricted use, distribution, and reproduction in any medium, provided the original work is properly cited.

\begin{abstract}
A three-dimensional numerical wave flume is employed to investigate the forces exerted by internal solitary waves (ISWs) on a pair of circular cylinders in tandem arrangement, using large-eddy simulation (LES) model. The effect of the centre-to-centre distance $(L)$ ranging from 1.5 to 5 diameters $(D)$ is studied for various ISWs amplitudes $\left(\eta_{a}\right)$ in the two-layer fluid system. Vertical-averaged vorticity distribution and vertical-averaged pressure gradient distribution in each layer are presented to investigate the different hydrodynamic interference between cylinders and the ISWs forces on each cylinder at various $L$. Furthermore, the force behaviors of the two cylinders are also compared with that of an isolated cylinder in the same environment. The interaction between the two piles occurs in both layers, and it is found that, for $1.5 \leq L / D<3.5$, strong mutual interference appears between two cylinders; for $3.5 \leq L / D<5$, the two cylinders continue to influence each other in a weak-interference state; for $L / D \geq 5$, the interaction gradually decreases into a noninteracting state. This paper tries to provide some references to structural arrangement of doublecylinder structure and grouped-cylinder structure in stratified flow environment.
\end{abstract}

\section{Introduction}

In oceans, estuaries, and lakes, the stable stratification of density happens while the fluid density changes along with the depth due to the variations of temperature, salinity, and other environmental factors. Internal solitary waves (ISWs) with different amplitudes may be produced by a tiny or weak perturbation in such stable stratified environment [1]. Based on the monitoring and measured data collected from the South China Sea, strong underwater currents caused by internal waves could be a serious threat to underwater structures, such as oil drilling platforms or supporting cylinders [2].

Thus more and more researchers got involved in the studies of IWs action on such structures. Laboratory experiment is an important approach to investigate the ISWs loading on cylinders. Ermanyuk and Gavrilov [3] experimentally studied the hydrodynamic loads exerted by ISW on a submerged circular cylinder in a two-layer system and discovered the locations of the maximum and minimum horizontal loads on the cylinder. Wei et al. [4] manufactured a new wave-maker to excite the ISWs and developed a measurement technique of hydrodynamic load to determine the interaction characteristics between IWs and an isolated cylindrical body in the tank.

Along with rapid development of computer and CFD technology, numerical methods have gotten more and more extensive application to investigate the loading induced by ISWs. The Morison empirical method [5], modal separation [6], and regression analyses [7] were applied to estimate the forces exerted by internal soliton on cylindrical piles. After that, a simplified estimation method of the force exerted by ISWs involving only the first mode internal wave was proposed [8]. The method can be used to estimate the force without observational current data and simplify the calculation procedure. For better solving the issues related to depression ISWs, an extended KdV model (EKdV) was employed to calculate the forces on a pile exerted by ISWs 
propagation from deep sea to shallow water [9]. Afterwards, $\mathrm{Si}$ et al. [10] used a generalized $\mathrm{KdV}$ model (GKdV) to obtain the vertical distribution of horizontal velocity of largeamplitude ISWs and discovered that the shear force was the largest at the turning point of the horizontal velocity. Based on water-wave theory, Linton and McIver [11] and Cadby and Linton [12] built a two-dimensional and three-dimensional numerical model, respectively, to study the interaction of waves with structures in two-layer fluids, and multipole expansions were used to solve the problems of wave radiation and scattering by a submerged structure in either the upper or lower layer. Then Sturova [13] conducted a study of radiation loads on interface piercing cylinder in a two-layer fluid of finite depth by a coupled element technique. By comparing with the ISWs force and the surface wave force, Du et al. [14] and Song et al. [15] found that the order magnitude of total force exerted by internal soliton was the same as that exerted by a surface wave, and the maximum total horizontal force caused by an internal wave amounted to $37.7 \%$ of that exerted by a surface wave [16]. Considering the above-mentioned, the ISWs force on underwater structures cannot be neglected.

Nevertheless, most researches before just focused on the cases of ISWs forces on an isolated cylindrical pile. In practical engineering, pile-supported structures are generally arranged in tandem or side by side with various centre-tocentre distance $(L)$ to form the so-called multiple slender structures, such as the bundle of risers linking the seabed to the offshore platforms and the piles being used for supporting bridges [17]. In general, two cylinders of the structures submerged in water behave in a similar manner to a single cylinder when the two cylinders are sufficiently apart. In some situations the cylinders need to be placed at a close proximity [18], and the interference between the two bodies significantly changes the flow around them [19]. Different flow patterns can be characterized by the behavior of the wake region [20], and unexpected flow structures and forces can be generated as the spacing between two circular cylinders changes [21].

Up to date, very few numerical investigations about the internal solitary waves (ISWs) action on a pair of circular cylinders in tandem arrangements can be found in the literatures. Thus, this paper aims to study numerically ISWs force behaviors of two tandem cylinders (placed parallel to wave direction). To begin with, large-eddy simulation model (LES) is employed to simulate the generation and propagation of depression ISWs in a three-dimensional numerical wave flume. With respect to two-phase stratified flow system, the horizontal current induced by ISWs reverses the flow direction between upper and lower layers. Therefore, the hydrodynamic interference occurring in each layer at diverse $L$ ranging from $1.5 D$ to $5 D$ will be investigated, respectively, from the perspective of vorticity distribution and pressure gradient distribution. The changes in vorticity fields and pressure gradient fields induced by different pile-to-pile interactions will be employed to study and explain the ISWs force behaviors of the two tandem cylinders. Finally, the results are also compared with that of an isolated cylinder in the same environment.

\section{Theoretical Foundation}

2.1. Korteweg-de Vries (KdV) Theory. Many nonlinear equations, represented by $\mathrm{KdV}$ equation, are widely applied to describe the propagation of the internal solitary waves in horizontal direction. KdV equation can be written as

$$
\frac{\partial \eta}{\partial t}+c_{0} \frac{\partial \eta}{\partial x}+\frac{3}{2} \frac{h_{1}-h_{2}}{h_{1} h_{2}} c_{0} \eta \frac{\partial \eta}{\partial x}+\frac{1}{6} h_{1} h_{2} c_{0} \frac{\partial^{3} \eta}{\partial x^{3}}=0,
$$

where $t$ is the time; $h_{1}$ and $h_{2}$ represent the thicknesses of the upper layer and the lower layer; the linear velocity of linear IWs $c_{o}$ and the interfacial vertical displacement $\eta$ can be, respectively, expressed as

$$
\begin{aligned}
& c_{o}=\sqrt{\frac{\left(g \Delta \rho h_{1} h_{2}\right)}{\rho_{2}\left(h_{1}+h_{2}\right)},} \\
& \eta=-\eta^{\prime} \operatorname{sech}^{2}\left(\frac{x-c_{p} t}{l}\right),
\end{aligned}
$$

where $\eta^{\prime}$ is the amplitude of incident wave; $c_{p}$ is the phase speed; $l$ is the characteristic wavelengths; $\Delta \rho$ is the density difference between two layers; $g$ is the gravity acceleration. $c_{p}$ and $l \mathrm{read}$

$$
\begin{aligned}
& c_{p}=c_{o}\left[1-\frac{\eta^{\prime}\left(h_{1}-h_{2}\right)}{2 h_{1} h_{2}}\right], \\
& l=\frac{2 h_{1} h_{2}}{\sqrt{3 \eta^{\prime}\left(h_{2}-h_{1}\right)}} .
\end{aligned}
$$

\section{Numerical Model}

3.1. Navier-Stokes Equations. The process of three-dimensional unsteady incompressible fluid motion governed by NavierStokes (N-S) equations can be described by

$$
\begin{gathered}
\frac{\partial u_{j}}{\partial x_{j}}=0 \\
\frac{\partial u_{i}}{\partial t}+u_{j} \frac{\partial u_{i}}{\partial x_{j}}=-\frac{1}{\rho} \frac{\partial p}{\partial x_{i}}+v \frac{\partial^{2} u_{i}}{\partial x_{j} \partial x_{j}}+f_{i},
\end{gathered}
$$

where $t$ is the time; $u_{j}$ is the velocity component; $x_{j}$ is the Cartesian coordinate; $p$ is the pressure; $v$ is the kinematic viscosity; and $f_{i}$ is the body force that equals the gravity acceleration $g$ in the vertical direction.

3.2. Scalar Transport Equation. The mass transfer between the two-layer water system has been taken into account. The scalar transport equation that governs the advectiondiffusion effect is as follows:

$$
\frac{\partial C}{\partial t}+u_{j} \frac{\partial C}{\partial x_{j}}=k \frac{\partial^{2} C}{\partial x_{j} \partial x_{j}}
$$

where $C$ is the volume concentration of brine water in the lower layer of the fluid system; $k$ is the molecular diffusivity coefficient. 


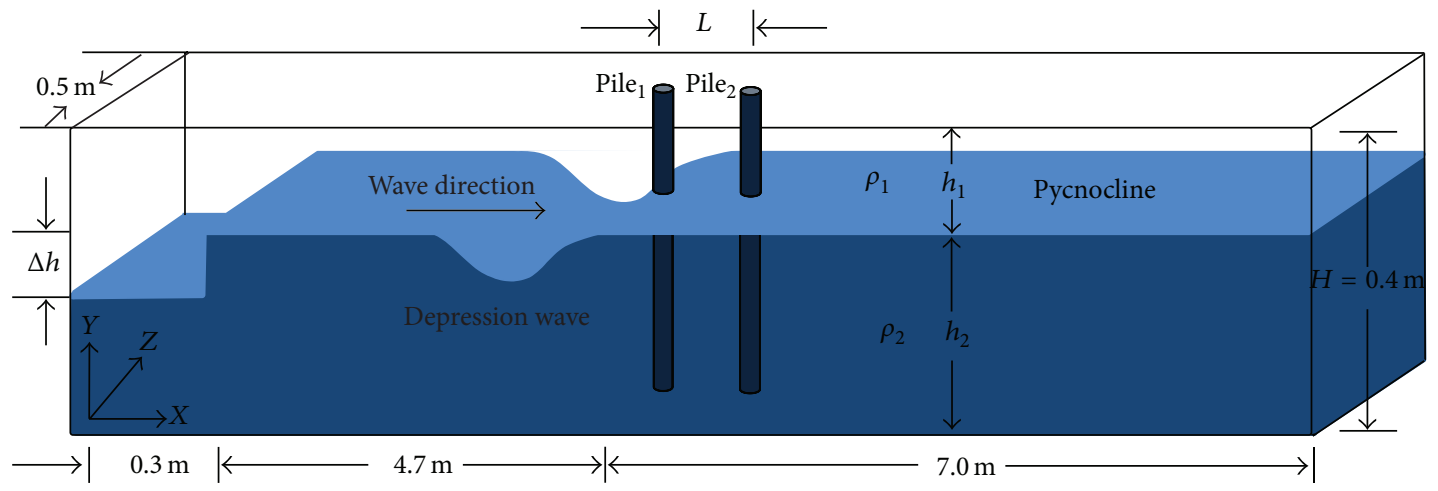

FIGURE 1: Schematic diagram showing the numerical model layout and the gravity collapse mechanism for ISW generation.

3.3. LES Governing Equations. Applying a spatial filter to Navier-Stokes equations, the filtered momentum and mass equations can be written as

$$
\begin{gathered}
\frac{\partial \overline{u_{j}}}{\partial x_{j}}=0 \\
\frac{\partial \overline{u_{i}}}{\partial t}+\overline{u_{j}} \frac{\partial \overline{u_{i}}}{\partial x_{j}}=-\frac{1}{\rho} \frac{\partial \bar{p}}{\partial x_{i}}+v \frac{\partial^{2} \overline{u_{i}}}{\partial x_{j} \partial x_{j}}+\frac{\partial \tau_{i j}}{\partial x_{i}}+\overline{f_{i}}, \\
\frac{\partial \bar{C}}{\partial t}+\overline{u_{j}} \frac{\partial \bar{C}}{\partial x_{j}}=k \frac{\partial^{2} \bar{C}}{\partial x_{j} \partial x_{j}}+\frac{\partial x_{j}}{\partial x_{j}},
\end{gathered}
$$

where the overbar notation denotes the application of tophat filter; the subgrid scale (SGS) stress tensor term $\tau_{i j}=$ $\overline{u_{i}} \overline{u_{j}}-\overline{u_{i} u_{j}}$ in (9) is responsible for the momentum exchange between the subgrid scale and the resolved scale; $\chi_{j}=\bar{C} \overline{u_{j}}-$ $\overline{\mathrm{Cu}}$ is the subgrid scalar flux responsible for the scalar flux exchange between the subgrid scale and the resolved scale. Hence, subsequent modeling is required to determine the turbulent viscosity $v_{t}$. As a function of the filter size and strain rate tensor, $v_{t}$ can be defined as

$$
v_{t}=\left(C_{s} \Delta\right)^{2}\left(2 \overline{S_{i j}} \overline{S_{i j}}\right)^{1 / 2}
$$

where $\overline{S_{i j}}=(1 / 2)\left(\partial^{2} \overline{u_{i}} / \partial x_{j}+\partial^{2} \overline{u_{j}} / \partial x_{j}\right)$ is the strain rate tensor; $C_{s}$ is the Smagorinsky constant; $\Delta$ is the filtered width. In general, the model coefficient $C_{s}$ varies in both time and space due to different flow conditions and even can be negative for a long time. Thus a dynamic procedure developed by Germano et al. [22] is employed to determine $C_{s}$.

3.4. Numerical Method and Boundary Condition. Large-eddy simulation model (LES) is employed to simulate the generation and propagation of ISWs of depression type. Velocitypressure term is solved by SIMPLE algorithm to enforce mass conservation and to obtain the pressure field, while secondorder centred differencing scheme is adopted for the spatial discretization, and the time step is discretized by secondorder implicit scheme.

The left boundary, belonging to the wave generation area where gravity collapse happens, and the two sidewalls and bottom of the wave tank are specified as a rigid wall with no-slip condition. Sommerfeld radiation type to avoid wave reflection is adopted to specify the right boundary, and the "rigid lid" approximation is used here to filter the free surface mode to ignore the influence of the surface wave [23].

3.5. Building of Numerical Wave Flume. A three-dimensional numerical wave flume established in current study is illustrated in Figure 1. The numerical wave tank in this paper has a dimension of $12 \mathrm{~m} \times 0.5 \mathrm{~m} \times 0.4 \mathrm{~m}$ in the streamwise $(X)$, span-wise $(Z)$, and vertical $(Y)$ direction, which represents the length, width, and height, respectively. This flume is divided into two parts, for one is the wave generation area which locates $L_{o}=0.3 \mathrm{~m}$ from the left boundary in the $X$ direction and the remaining is the wave propagation area. The two piles are placed in tandem arrangement. The bottom centre of the upstream cylindrical pile (Pile ${ }_{1}$ ) locates at $(x, y, z)=(5,0,0.25)$ from the coordinate origin, while $L$ is the centre-to-centre distance between Pile $_{1}$ and the downstream cylindrical pile $\left(\right.$ Pile $\left._{2}\right) . D$ is the diameter of two piles. The density is $\rho$, and the thickness of each layer is $h$, of which the subscripts 1 and 2 represent the upper layer and the lower layer. The upper-layer fluid density $\rho_{1}$ is set to be $1000 \mathrm{~kg} / \mathrm{m}^{3}$, the lower-layer fluid density $\rho_{2}$ is set to be $1030 \mathrm{~kg} / \mathrm{m}^{3}$, and the volumetric concentration $C$ of the brine water in the lower layer is around 3\%. The step height $\Delta h$ we called here is the height difference of the pycnocline. The whole two-layer system keeps quiescent at the initial time.

Gravity collapse in a two-layer stratified fluid system is adopted to excite the ISWs [24]. Compared with the method of oscillating boundary or the movable body, the ISWs generation method of gravity collapse is simpler and widely employed in the laboratory experiments and numerical simulations [25]. An ISW of depression type forms here by making the height of the pycnocline in the wave propagation area higher than the middle depth, as shown in Figure 1.

3.6. Verification of the Numerical Model. The KdV equation is employed to validate the waveform firstly, then followed by a grid-size convergence analysis. The numerical simulation results of ISWs forces on an isolated pile are verified finally by comparing with the experiments in laboratory and the Morison equation. 


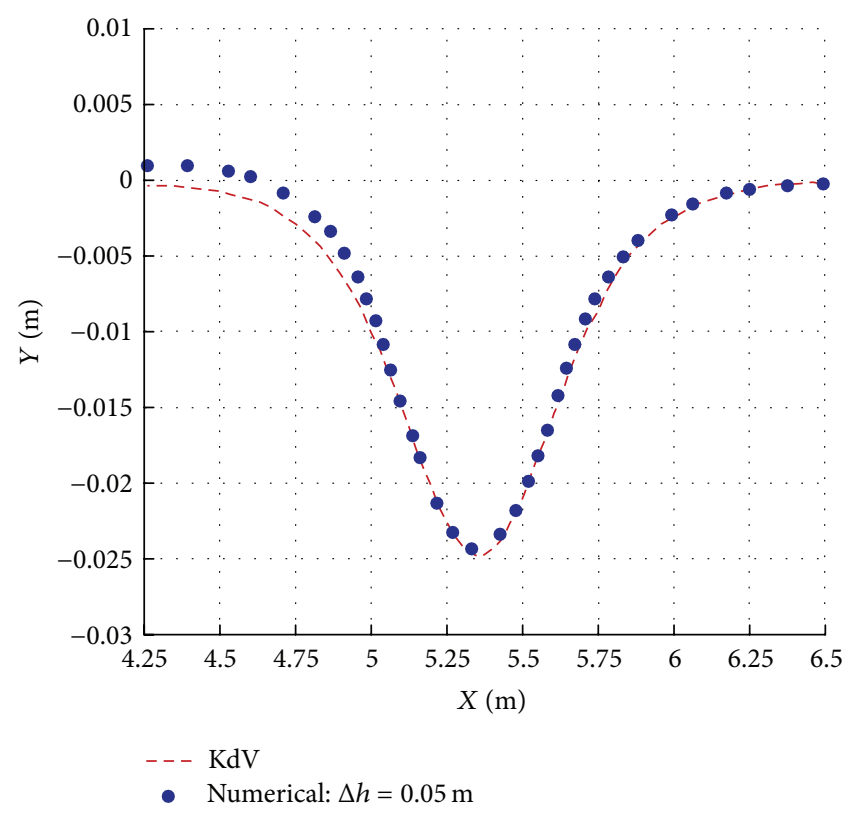

(a)

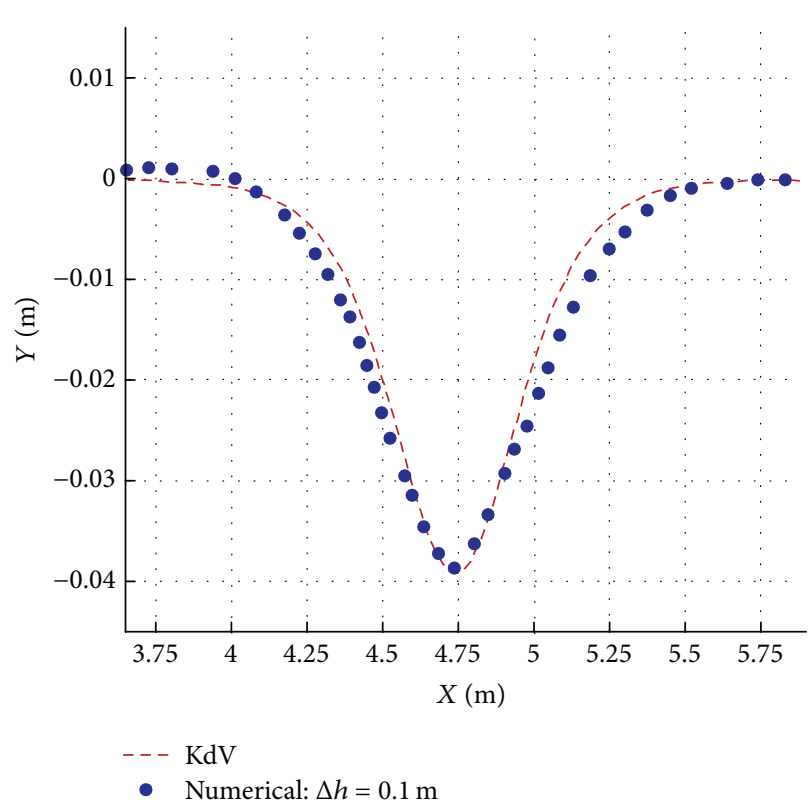

(b)

FIGURE 2: Comparison of numerical results with KdV solutions for wave profiles: (a) A1; (b) A2.

TABLE 1: Corresponding parameters of waveform verification cases.

\begin{tabular}{lccccc}
\hline Simulation number & Wave type & $\Delta h$ & $h_{1}(\mathrm{~m})$ & $h_{2}(\mathrm{~m})$ & $\eta_{o} / H$ \\
\hline $\mathrm{A} 1$ & Depression & 0.05 & 0.1 & 0.3 & 0.062 \\
$\mathrm{~A} 2$ & Depression & 0.1 & 0.1 & 0.3 & 0.0985 \\
\hline
\end{tabular}

3.6.1. Verification of Wave Profiles. Two cases of waveform, with different wave amplitudes $\eta_{o}$, simulated by the numerical model have been compared with the KdV solutions. The corresponding parameters are shown in Table 1 , where $\Delta h$ is the step height, $\eta_{o}$ is the amplitude of an ISW, and $H=0.4 \mathrm{~m}$ is the total water depth in the tank.

The total simulation time is $50 \mathrm{~s}$ for both verification cases and wave profiles are extracted at $t=33 \mathrm{~s}$. Verification results show that the numerical simulations are in good agreement with the KdV equation, and waveform of smaller amplitude fits better with the solutions (see Figure 2).

3.6.2. Grid-Size Convergence Analysis. To assess the reliability of the proposed results, a grid independence study carried out on three different grids for an isolated circular cylinder is performed below. The difference in the grids is principally based on the number of nodes on the cylinder circumference and the total nodes in the $Y$ direction. Details of the grid independence test are given in Table 2, where $\eta_{a} / H$ is the nondimension amplitude, $\Delta t$ is the time step, and $C_{F_{x \max }}$ represents the dimensionless global horizontal force amplitude exerted by internal solitary waves. The time step $\Delta t$ can be chosen by the following expression [26]: $\mathrm{Cr}=c_{p} \Delta t / \Delta x<1$, $\Delta t<\Delta x / c_{p} \approx 0.02$, where $\mathrm{Cr}$ is the Courant number, $c_{p}$ is the phase speed of internal solitary wave, and $\Delta x$ is the minimum grid length in the $X$ direction. Finally, we selected the time step $\Delta t=0.01 \mathrm{~s}$ in this paper. Close-up views of the triangular unstructured mesh around an isolated cylinder of the three meshes are illustrated in Figure 3.

Figure 4 shows the computational results of three different mesh densities. It is observed that an increase in resolution from case $T_{1}$ (low) to case $T_{2}$ (moderate) gave an obvious difference in the magnitude of $C_{F_{x}}$; however, by comparing case $\mathrm{T}_{2}$ with case $\mathrm{T}_{3}$ (high), the curves of the nondimensional ISWs forces show that the difference between the two computations is very small. Hence, the computations are considered grid independent.

3.6.3. Verification of Forces Acting on an Isolated Circular Pile. The numerical simulation results of ISWs forces on an isolated pile are verified by Morison equation and the experiments in laboratory.

(1) Verification by Morison Equation. The Morison equation [27] can be adopted if the diameter of a pile, $D$, is supposed to be less than the wave length, $L_{o}, D / L_{o} \leq 0.15$. In this investigation, the diameter of pile, $D=0.05 \mathrm{~m}$, is much smaller than the leading soliton length, $L_{o} \approx 2 \mathrm{~m}$, making $D / L_{o} \leq 0.15$ become satisfied.

The global force $F_{m}$, made up of a drag component $F_{D}$ and an inertial component $F_{I}$, on a cylindrical pile can be expressed as

$$
F_{m}=F_{D}+F_{I} .
$$

$F_{D}$ and $F_{I}$ are determined by the following equations, respectively [14]:

$$
\begin{aligned}
F_{D} & =\frac{1}{2} \int_{0}^{H} \rho g C_{D} D u|u| d z, \\
F_{I} & =\int_{0}^{H} \rho g C_{M} \frac{\pi D^{2}}{4} \frac{\partial u}{\partial t} d z
\end{aligned}
$$


TABLE 2: Details of the grid independence test carried out at $\eta_{a} / H=0.215$.

\begin{tabular}{|c|c|c|c|c|}
\hline Test number & Number of nodes on the cylinder circumference & Number of nodes in the vertical direction & $\Delta t(\mathrm{~s})$ & $C_{F_{x \max }}$ \\
\hline $\mathrm{T}_{1}$ (low) & 30 & 33 & 0.02 & 1.899 \\
\hline $\mathrm{T}_{2}$ (moderate) & 60 & 65 & 0.01 & 2.258 \\
\hline $\mathrm{T}_{3}$ (high) & 90 & 98 & 0.006 & 2.298 \\
\hline
\end{tabular}

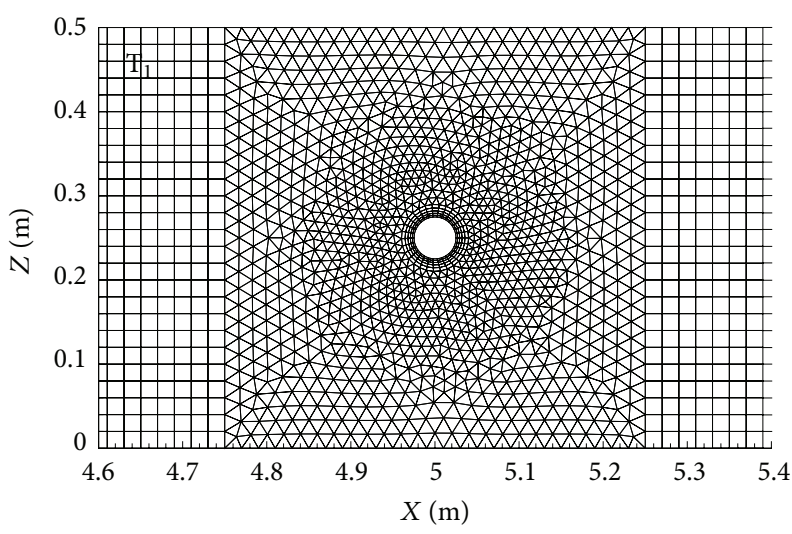

(a)

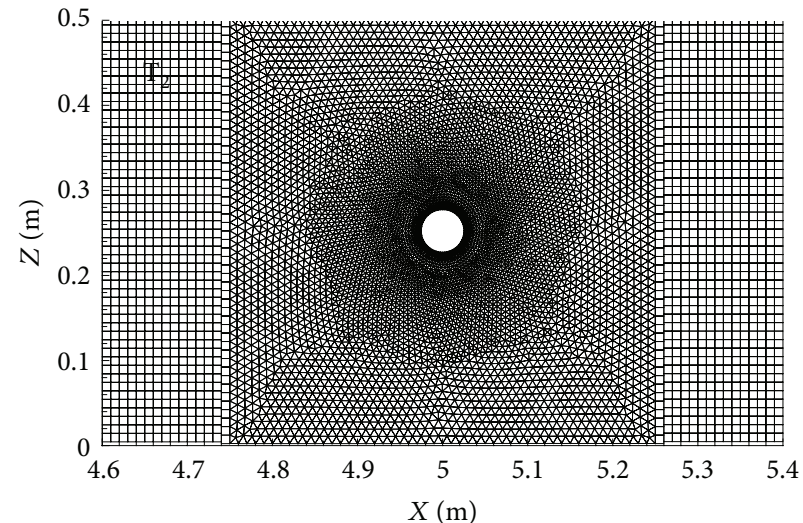

(b)

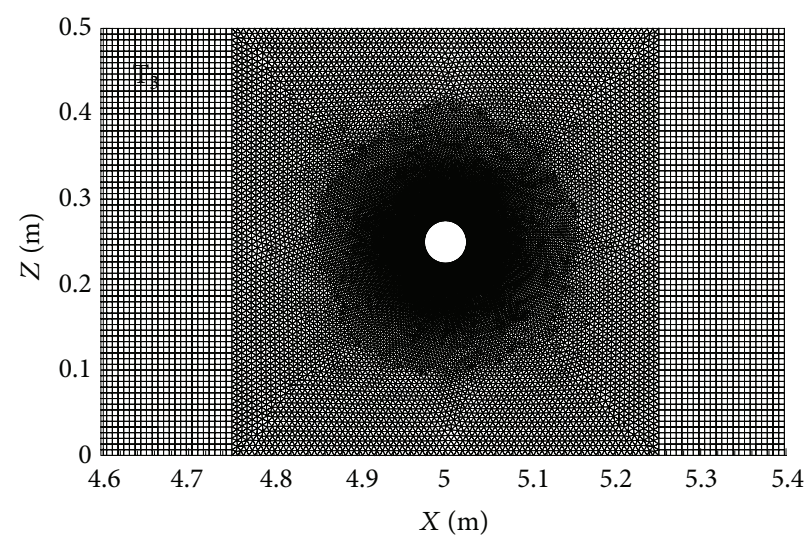

(c)

FIGURE 3: Close-up view of the triangular unstructured mesh around a single circular cylinder: (a) $\mathrm{T}_{1}$ : low density; (b) $\mathrm{T}_{2}$ : moderate density; (c) $\mathrm{T}_{3}$ : high density.

where $\rho$ is water density, $g$ is gravity acceleration, $D$ is the diameter of pile, $u$ is the horizontal component of the current velocity, $t$ is the time, $z$ is the depth, and $H$ is the total water depth in the tank. $C_{D}$ is the drag-force coefficient, and $C_{M}$ is the inertia-force coefficient. The values of $C_{D}$ and $C_{M}$ over a wave cycle vary with the current intensity and the size of the cylinders. The two coefficients, correlated with the Keulegan-Carpenter number (KC) and the Reynolds number (Re), can be determined experimentally [28]. In this paper, $\mathrm{KC}=u_{\max } T / D=26.5, \operatorname{Re}=u_{\max } D / \nu=12000$, where $u_{\max }$ is the maximum internal wave-induced velocity, $T$ is the period of the internal wave, $D$ is the diameter of the pile, and $v$ is the kinematic viscosity of fluid. According to the experimental results of Sarpkaya and Michael [29], $C_{D}=0.6, C_{M}=1.8$ are chosen in the calculation.
(2) Verification by Experiments. Beyond the analysis performed by Morison equation, further validation of the numerical method is added by comparison with laboratory experiments.

The experiments of ISWs force measurement are conducted in a long stratified fluid tank at the PLA University of Science and Technology, which is made of steel frame and glass materials with the dimension of $12 \mathrm{~m}$ length, $0.5 \mathrm{~m}$ width, and $0.5 \mathrm{~m}$ depth, as shown in Figure 5. The experiment is implemented with initial conditions as close to those in the numerical setup as possible: the total water depth $H$ in the tank is $0.4 \mathrm{~m}$, while $h_{1}=0.1 \mathrm{~m}, h_{2}=0.3 \mathrm{~m}, \rho_{1}=1000 \mathrm{~kg} / \mathrm{m}^{3}$, $\rho_{2}=1030 \mathrm{~kg} / \mathrm{m}^{3}$, and the density difference $\Delta \rho$ between the two layers is set to be $30 \mathrm{~kg} / \mathrm{m}^{3}$. Referring to the wavegenerating method used in numerical simulation, gravity 


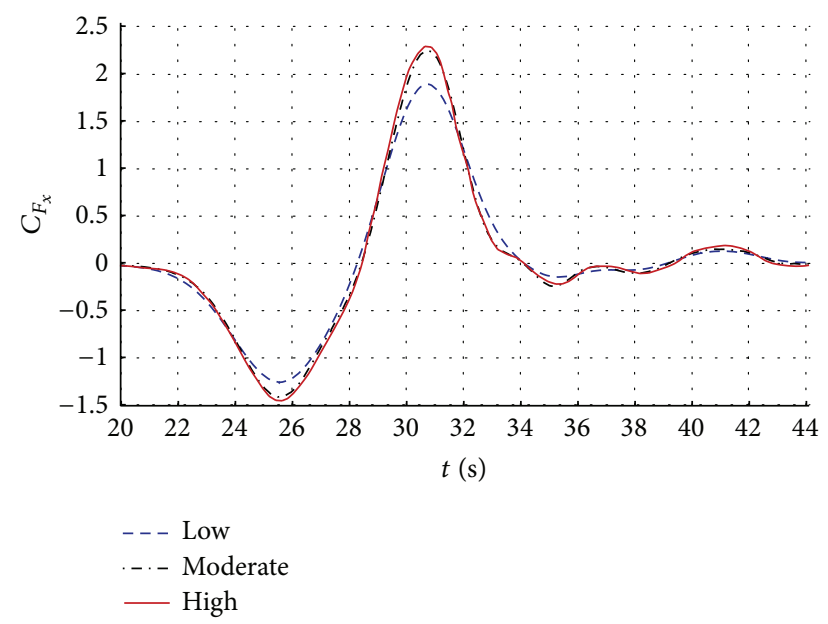

FIgURE 4: Computational results of three different mesh densities, carried out at $\eta_{a} / H=0.21$.

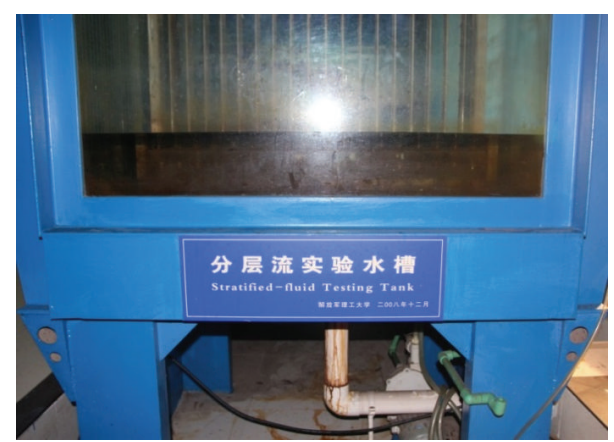

FIGURE 5: Schematic diagram of the stratified fluid test tank.

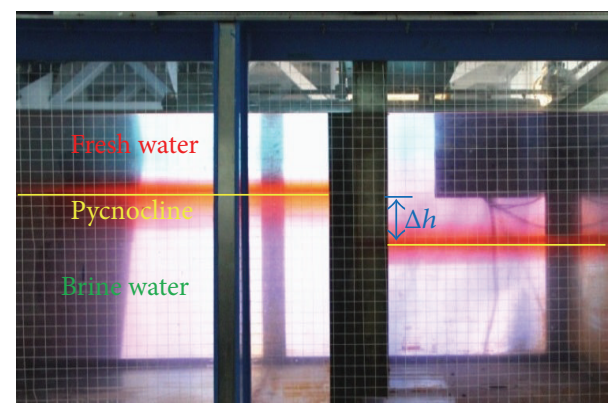

FIgURE 6: Snapshot of the gravity collapse mechanism for ISW generation at the initial time.

collapse in a two-layer stratified fluid system is adopted to excite the ISWs in the experiments. The initial time of gravity collapse in the tank is shown in Figure 6.

A slender cylinder is of $0.4 \mathrm{~m}$ length and $0.05 \mathrm{~m}$ diameter, the size of which is the same as the one used in the numerical simulation. The cylinder is placed at the centre of the tank in the span-wise direction and a distance of 5 meters from the right side. A force-measuring sensor with a small range and high precision and sensitivity is used to measure the force exerted by internal waves on the model. The sensor is connected to the test cylinder through an aluminum slender
TABLE 3: Corresponding parameters of force verification cases.

\begin{tabular}{lccccc}
\hline Simulation number & Wave type & $\Delta h(\mathrm{~m})$ & $h_{1}(\mathrm{~m})$ & $h_{2}(\mathrm{~m})$ & $\eta_{a} / H$ \\
\hline B1 & Depression & 0.15 & 0.1 & 0.3 & 0.183 \\
B2 & Depression & 0.2 & 0.1 & 0.3 & 0.215 \\
\hline
\end{tabular}

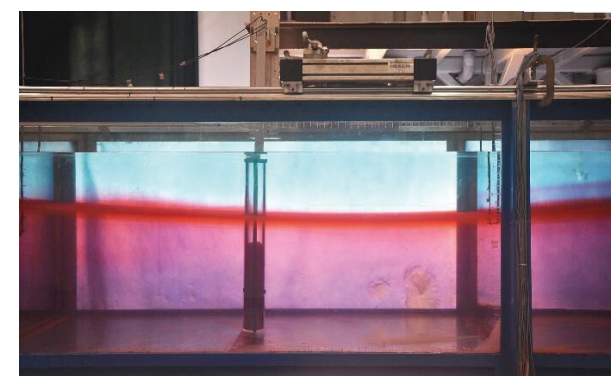

FIGURE 7: Photo of the force measurement setup and ISW acting on the cylinder.

rod functioning as force transferring portion of the system; see Figure 7. Then records of ISWs forces $F_{e}$ versus time of different step heights and ISWs amplitudes can be obtained by the Spider8 processor made in Germany and the special software, which was of good reliability, high precision, and rapid speed. The principle of generating internal solitons and measuring forces in a stratified fluid tank is shown in Figure 8.

Three nondimensional formulas of force are defined as follows:

$$
\begin{aligned}
C_{F_{x}} & =\frac{2 F_{x}}{\rho u_{\text {max }}^{2} D H}, \\
C_{F_{m}} & =\frac{2 F_{m}}{\rho u_{\text {max }}^{2} D H}, \\
C_{F_{e}} & =\frac{2 F_{e}}{\rho u_{\max }^{2} D H},
\end{aligned}
$$

where $F_{x}$ denotes the global horizontal force calculated by the numerical model, $F_{m}$ denotes the global horizontal force calculated by the Morison equation, $F_{e}$ denotes the global horizontal force obtained by experiments, and $u_{\max }$ represents the maximum internal wave-induced velocity (positive in the direction of wave propagation from left to right when facing the wave flume). The computing time for each verification case is $50 \mathrm{~s}$, and the corresponding parameters of two force verification cases are listed in Table 3 , where $\eta_{a}$ is the amplitude when the leading ISW just reaches the pile.

The comparisons of numerical simulations with experiments and Morison equation for ISWs forces $C_{F}$ on the isolated pile versus time are given in Figure 9. A similar overall trend among the three approaches can be observed in the curves, and the experimental results $C_{F_{e}}$ agree better with the numerical results $C_{F_{x}}$ than that of Morison equation $C_{F_{m}}$. It should be pointed out that the positive direction of $C_{F}$ defined in this paper is along the direction of ISWs propagation from left to right.

From the above three aspects (waveform, grid-size convergence, and wave force) of verification, it can be concluded 


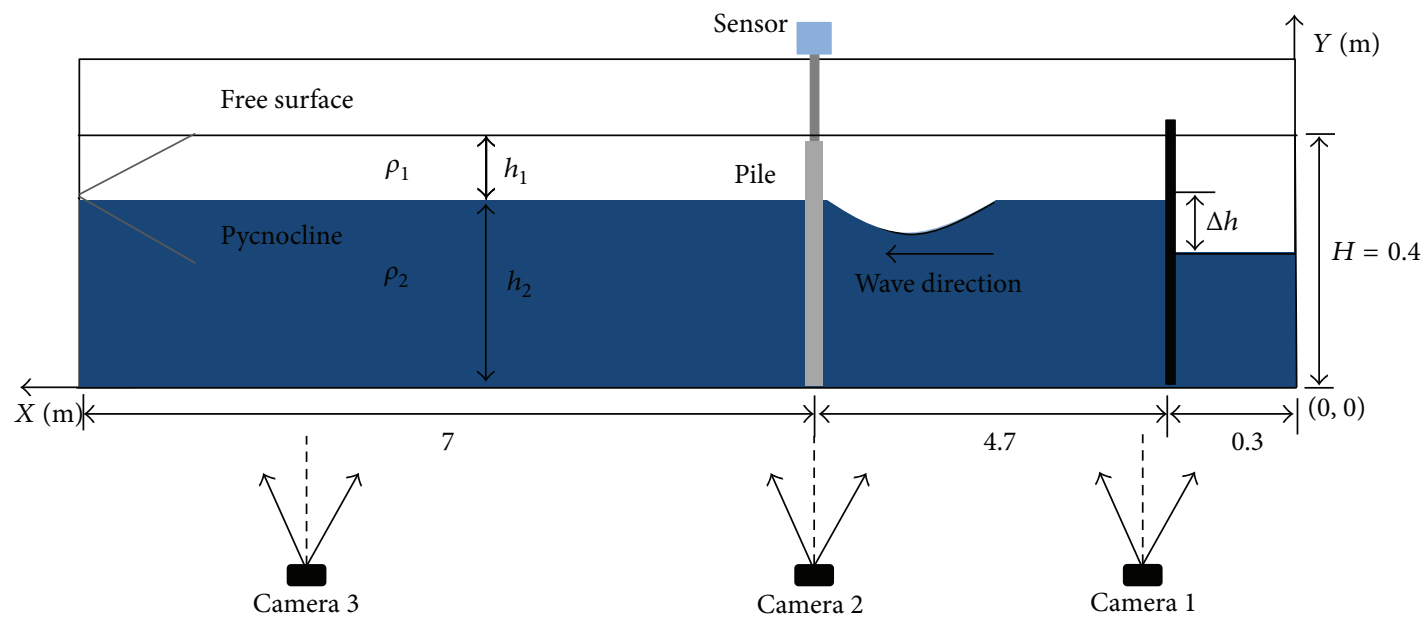

FIGURE 8: Schematic diagram of generating internal solitons and measuring forces in a stratified fluid tank.

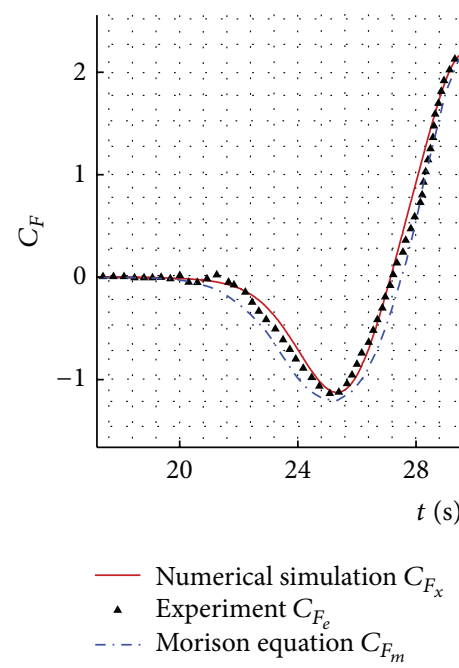

(a)

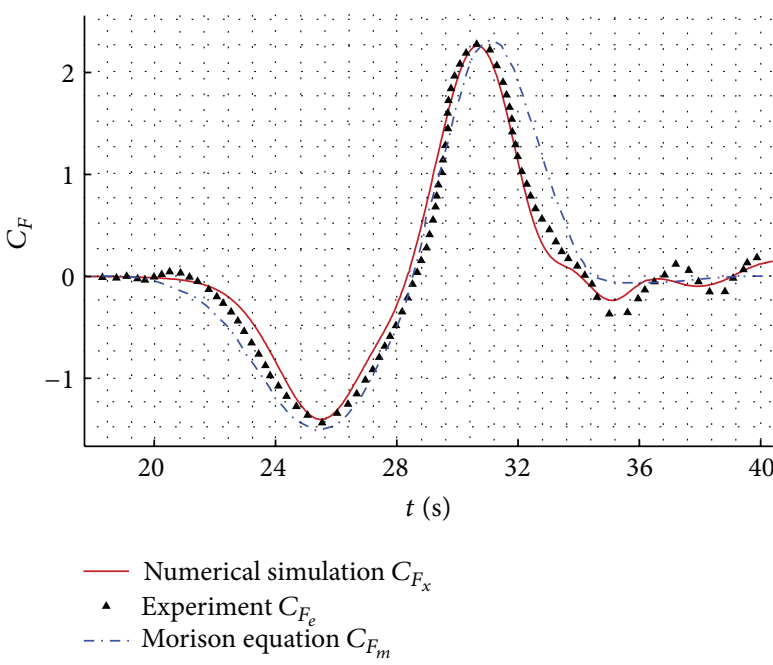

(b)

FIgURE 9: Comparison of numerical simulations with experiments and Morison equation for ISWs forces on an isolated pile: $(\mathrm{a}) \mathrm{B1}: \eta_{a} / H=$ 0.1825 ; (b) B2: $\eta_{a} / H=0.215$.

that the numerical model is reliable to simulate the generation and propagation of ISWs of depression type and capable of calculating the force on a cylinder exerted by ISWs.

\section{Results and Discussion}

The flow field largely depends on Reynolds number, range of which covers $7800 \leq \operatorname{Re}=u_{\max } D / v \leq 12000$ for different dimensionless ISWs amplitudes $\eta_{a} / H$ ranging from 0.12 to 0.215 , when it comes to flow around a single circular cylinder [30], while as to the case of two tandem cylinders, the dimensionless centre-to-centre distance $L / D$ also plays a decisive role [31, 32]. In order to investigate the forces on a multiple slender structure and the hydrodynamic interference between the cylinders, simulations have been carried out for two cylinders in tandem arrangements.
To begin with, note that in single-layer flow environment, flows around two circular cylinders in tandem arrangements can be grouped into two main categories: with or without mutual interference [22]. Furthermore, different interference regions in the "mutual interference" category were identified by the classification of Igarashi [33]. Then the pressure and forces on two tandem cylinders in these regions can be analyzed by employing the vortex method [34].

With respect to two-phase stratified flow system, as seen in Figure 10, while the direction of the ISWs propagation is from left to right, the horizontal current induced by internal waves reverses the flow direction between upper and lower layers. The interaction between Pile ${ }_{1}$ and Pile ${ }_{2}$ occurs in both layers. Therefore, the physical mechanisms of the interference between the two cylinders should be studied separately in each layer, which acts as an important method contributing 
TABLE 4: Typical simulation conditions.

\begin{tabular}{|c|c|c|c|c|}
\hline Simulation number & Wave type & $\Delta h(\mathrm{~m})$ & $\eta_{a} / H$ & $L / D$ \\
\hline $\mathrm{Cl}$ & Depression & 0.1 & 0.12 & 1.5 \\
\hline $\mathrm{C} 2$ & Depression & 0.2 & 0.215 & 1.5 \\
\hline $\mathrm{C} 3$ & Depression & 0.2 & 0.215 & 2 \\
\hline $\mathrm{C} 4$ & Depression & 0.2 & 0.215 & 2.5 \\
\hline $\mathrm{C} 5$ & Depression & 0.2 & 0.215 & 3.5 \\
\hline C6 & Depression & 0.2 & 0.215 & 5 \\
\hline
\end{tabular}

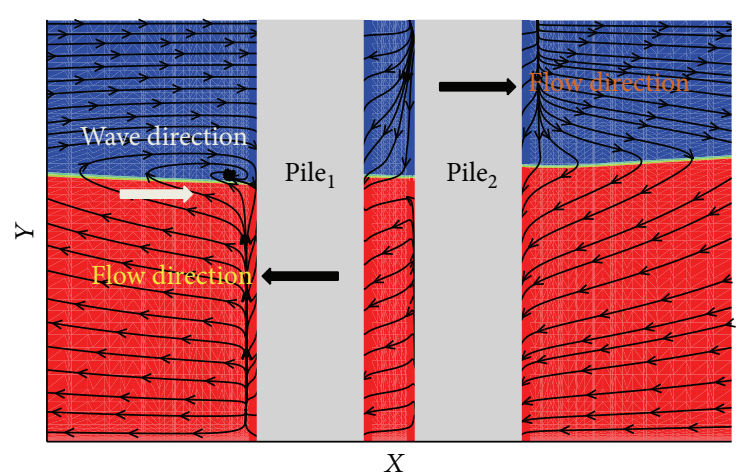

FIGURE 10: Instantaneous streamlines profiles showing the wave direction of two layers.

to investigating the direction and the effect of ISWs loading on a multiple slender structure.

Totally 45 cases are simulated to investigate the ISWs forces on two tandem piles for the range of $\Delta h$ from $0.1 \mathrm{~m}$ to $0.2 \mathrm{~m}$ and $L / D$ from 1.5 to 5 . The typical simulation conditions of $\Delta h=0.2$ are listed in Table 4 , where $\eta_{a}$ is the wave amplitude when the leading ISW just reaches Pile $_{1}$. The hydrodynamic interference induced by ISWs occurring in each layer at diverse $L$ will be, respectively, discussed in detail below from the perspective of vorticity distribution and pressure gradient distribution [21]. The analysis of flow field characteristic and the forces of Pile ${ }_{1}$ will be discussed in Sections 4.1 and 4.2 and then followed by those of Pile 2 in Sections 4.3 and 4.4.

4.1. Analysis of the Flow Field Characteristic around Pile for Various Tandem Arrangements. (a) At the gap of 1.5D: in the upper layer, the plots in Figure 11 show the contours of instantaneous vertical-averaged pressure gradient distribution of the two tandem piles and the isolated pile in the upper layer, where Zone $\mathrm{A}$ and Zone $\mathrm{B}$ denote the favorable pressure gradient area and the adverse pressure gradient area (all the instantaneous contours shown in this paper are adopted when $C_{F_{x}}$ reaches its maximum for each simulation case [4]). By comparing the contours of $L / D=1.5$ in Figure 11(a) with that of an isolated pile in Figure 11(b), one can clearly observe how the adverse pressure gradient (Zone $\mathrm{B}$ ) behind Pile ${ }_{1}$ is influenced and suppressed by the presence of the downstream body at the gap of $1.5 \mathrm{D}$; in the lower layer, Figure 12(a) shows the instantaneous vertical-averaged vorticity contours with streamlines. Contours are extracted when $C_{F_{x}}$ is the maximum. It is important to note that $\mathrm{Pile}_{1}$ is inside the near wake and immersed in a low-pressure region formed by the separated shear layers emanating from $\mathrm{Pile}_{2}$.

(b) At the gap of $2 D$ : in the upper layer, Zone A and Zone $\mathrm{B}$ of $L / D=2$ shown in Figure 11(c) are very similar to those in Figure 11(b). It indicates that the effect of pressure gradient on Pile ${ }_{1}$ will not work when the gap is greater than $2 D$. Thus only the vortex effect in the lower layer is needed to be given consideration for gaps $\geq 2 D$ for Pile ${ }_{1}$; in the lower layer, as illustrated in Figure 12(b), the rear of Pile 1 begins to be hit by the vortices emanated from Pile $_{2}$ instead of being immersed in the low-pressure region formed in front of Pile ${ }_{2}$ [35].

(c) At the gap of $3.5 \mathrm{D}$ : in the lower layer, vortices generated in front of $\mathrm{Pile}_{2}$ continue to influence the horizontal forces on Pile $_{1}$ after the strong mutual interference region (spacing $\leq 2 D$ ); see Figure 12(c). The impact force exerted by vortices decreases with the increase of the gap.

(d) At the gap of 5D: in the lower layer, the interference between the two piles gradually decays into a noninteracting state presenting between the two piles in Figure 12(d).

Therefore, on the basis of the above analysis, the interference regions between two cylinders for $\mathrm{Pile}_{1}$ can be preliminarily classified into three interference regions: $1.5 \leq$ $L / D<2,2 \leq L / D<3.5$, and $3.5 \leq L / D \leq 5$. And the changes of vorticity fields and pressure gradient fields induced by different hydrodynamic interactions in these regions will be employed to study and explain the ISWs force behaviors of Pile $_{1}$ in the following.

4.2. ISWs Forces on Pile 1 . Curves of $C_{F_{x \max }}$ changing with $L / D$ for different $\eta_{a} / H$ of Pile ${ }_{1}$ are shown in Figure 13 , where $C_{F_{x \max }}$ represents the dimensionless global horizontal force amplitude for each simulation case, and $\eta_{a} / H$ denotes the dimensionless wave amplitude. First of all, it should be noted that the positive direction of all forces defined in this study coincides with the direction of ISWs propagation from left to right, and the negative direction is from right to left. Besides, two physical quantities $f_{1}$ and $f_{2}$ are defined here to simplify the descriptions of the interaction force between two piles, as illustrated in Figure 14, where $f_{1}$ denotes the horizontal force acting on the rear of Pile $e_{1}$ in the negative direction; $f_{2}$ denotes the horizontal force acting on the front of $\mathrm{Pile}_{2}$ in the positive direction.

As it can be seen in Figure 13, the overall trends of all the five curves of $C_{F_{x \max }}$ on Pile $_{1}$ are the same in respect to the variations of $L / D$. Below the spacing of $2 D$, it can be found that $C_{F_{x \max }}$ acting on Pile $_{1}$ reaches its minimum at the gap of $2 D$ after experiencing the maximum at the gap of $1.5 D$. 

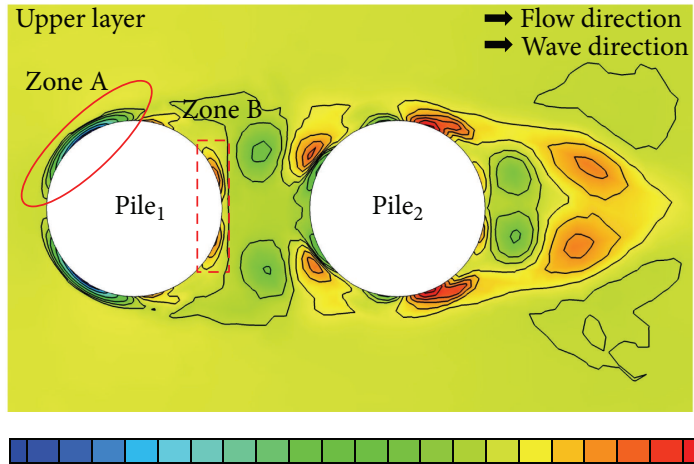

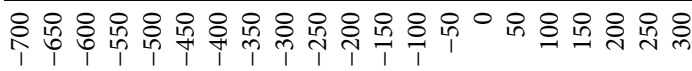

(a)
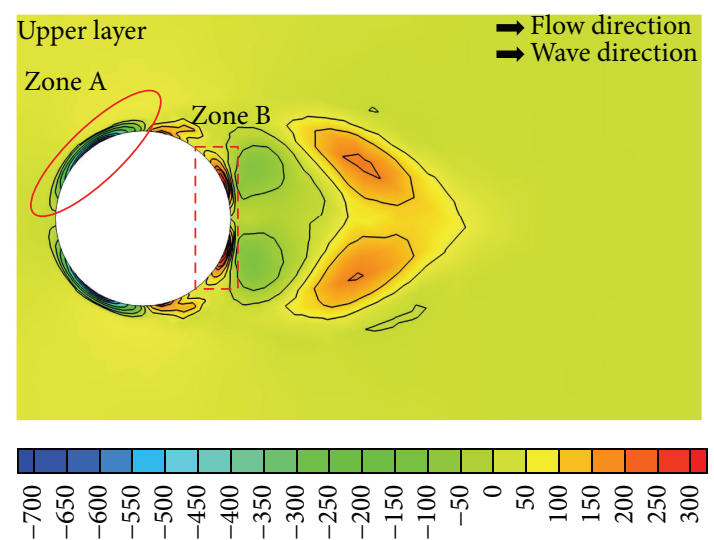

(b)

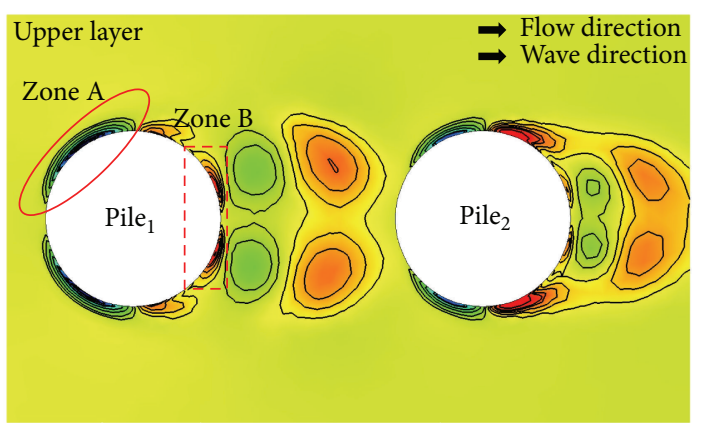

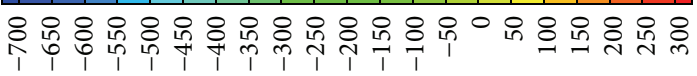

(c)

FIGURE 11: Instantaneous vertical-averaged pressure gradient contours when $C_{F_{x}}$ is the maximum, $\eta_{a} / H=0.215$ : (a) $L / D=1.5$, (b) isolated pile, and (c) $L / D=2$, in the upper layer.

At the gap of $1.5 \mathrm{D}$ in the upper layer, the drop of the adverse pressure gradient (by comparing Figure 11(a) with Figure 11(b)) reduces $f_{1}$, enlarging $C_{F_{x}}$ as well as its amplitude $C_{F_{x \max }}$; meanwhile, in the lower layer, the low-pressure region formed in front of Pile $_{2}$ also reduces $f_{1}$ [20]. So $C_{F_{x \max }}$ increases in both layers and meets its maximum at $1.5 \mathrm{D}$.

At the gap of $2 D$, the influence of the pressure gradient on $C_{F_{x \max }}$ (mentioned in Section 4.1(b)) being ignored, only the vortex effect in the lower layer should be taken into account. As illustrated in Figure 12(b), Pile ${ }_{1}$ is no longer influenced by the low-pressure region and turns to be hit by the impact force exerted by vortices (in the negative direction), making $f_{1}$ larger. So $C_{F_{x \max }}$ will be decreased by being counteracted by $f_{1}$ and thus reach its valley value at this gap.

In the next stage when the intervals are in the range of $2<$ $L / D<3.5$, the curves of all the five configurations present a positive slope; see in Figure 13. A continued growth of $C_{F_{x \max }}$ can be found in this region. It is because the impact force of vortices on the rear of Pile ${ }_{1}$ keeps on reducing as the distance increases further.

If the gap is gradually increased to $5 D$, further increase of $\mathrm{L}$ brings little change of $C_{F_{x \max }}$ above the spacing of $3.5 \mathrm{D}$ for all configurations. For a better understanding, $C_{F_{x}}$ changing with $\Delta h / H$ of the two tandem piles is compared with that of an isolated cylinder at this gap, as shown in Figure 15(c), where the forces on the two tandem cylinders are in a similar way to that on the single cylinder. It is indicated that the two-pile interaction will gradually disappear when $L / D>5$.

A point worth noting is that a shape of "kink" can be found below the gap of $2.5 D$, as illustrated in Figure 13, and the "kink" becomes more pronounced as $\eta_{a} / H$ gets larger. See Line 1 in Figure 15(a); the trend graph of $C_{F_{x \max }}$ with $\eta_{a} / H$ shows that the increase of ISWs amplitude leads to a rapid rise in horizontal force on upstream pile at the gap of $1.5 D$. It can be explained by the fact that the vortex intensity between two piles for the configuration of $\eta_{a} / H=0.215$ is significantly stronger than that of $\eta_{a} / H=0.12$, evidenced by comparing the vorticity contours in Figure 16(a) with that in Figure 16(b). The comparison of the two figures also reveals that the case of $\eta_{a} / H=0.215$ can provide clearer contours diagrams for visualizing when compared with the case of smaller wave amplitude. Since the forces trend curves of Pile show a similar behavior, the configuration of $\Delta h=0.2 \mathrm{~m}$ $\left(\eta_{a} / H=0.215\right)$ can be taken as a typical condition to elaborate and analyze the force behaviors of piles here. The contours of other $\eta_{a} / H$ configurations will not be given in this paper. 


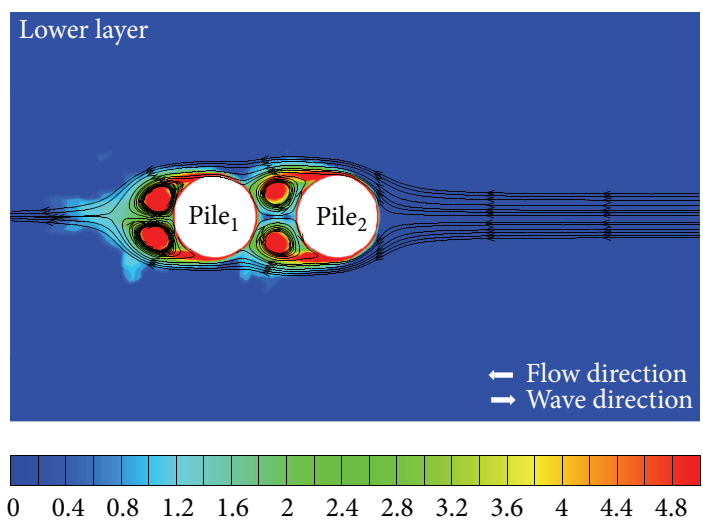

(a)

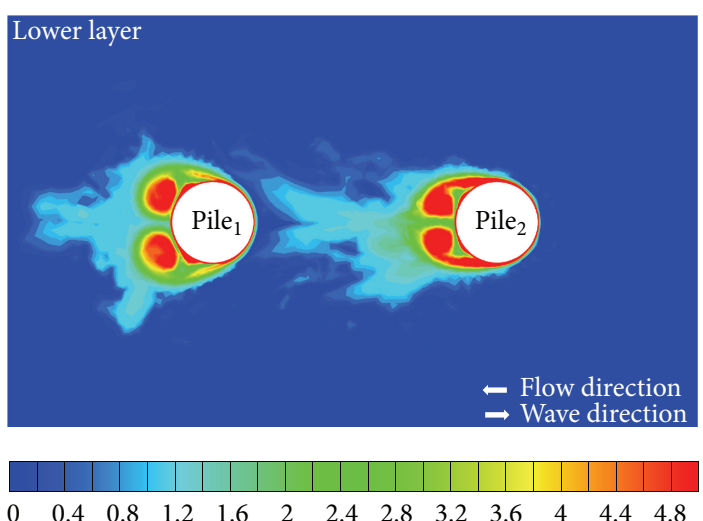

(c)

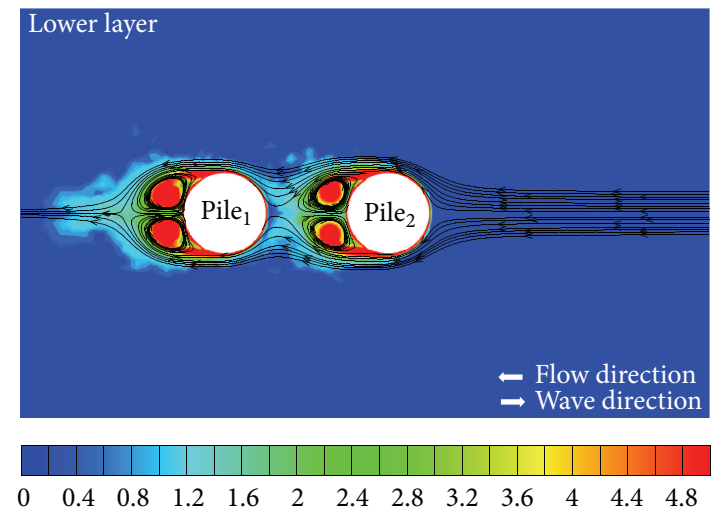

(b)

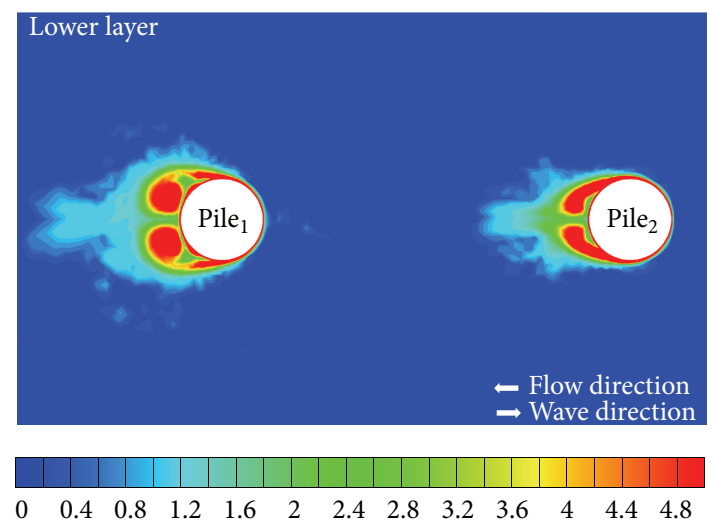

(d)

FIGURE 12: Instantaneous vertical-averaged vorticity contours and streamlines for diverse $L / D$ when $C_{F_{x}}$ is the maximum, $\eta_{a} / H=0.215:(a)$ $L / D=1.5$, (b) $L / D=2$, (c) $L / D=3.5$, and (d) $L / D=5$, in the lower layer.

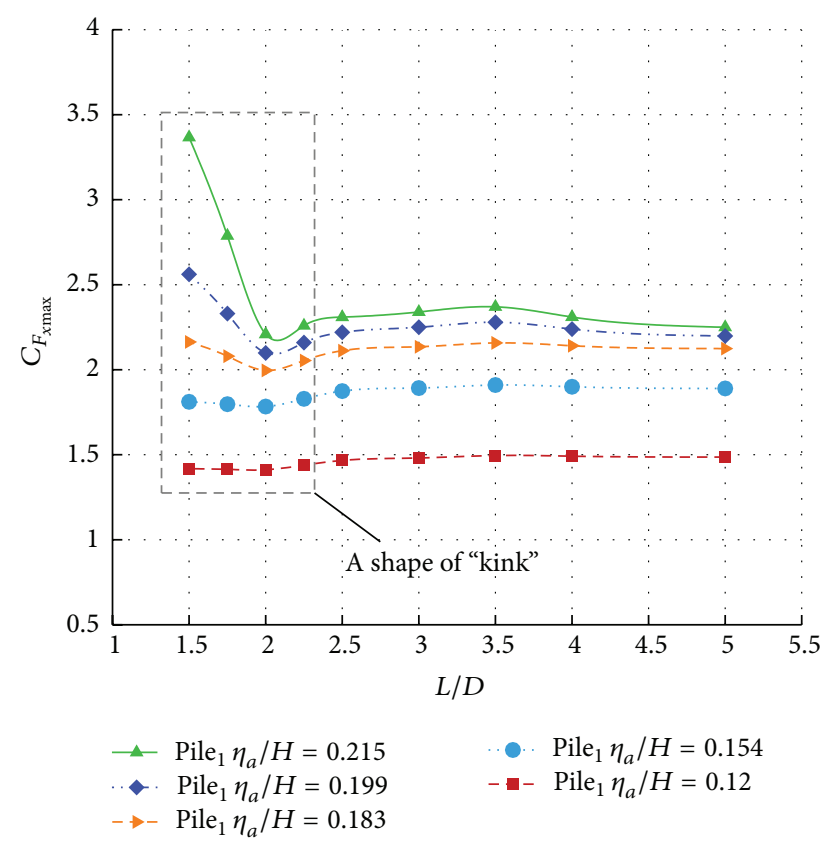

FIGURE 13: Variations of $C_{F_{x \max }}$ with $L / D$ for different $\eta_{a} / H$ of Pile ${ }_{1}$.

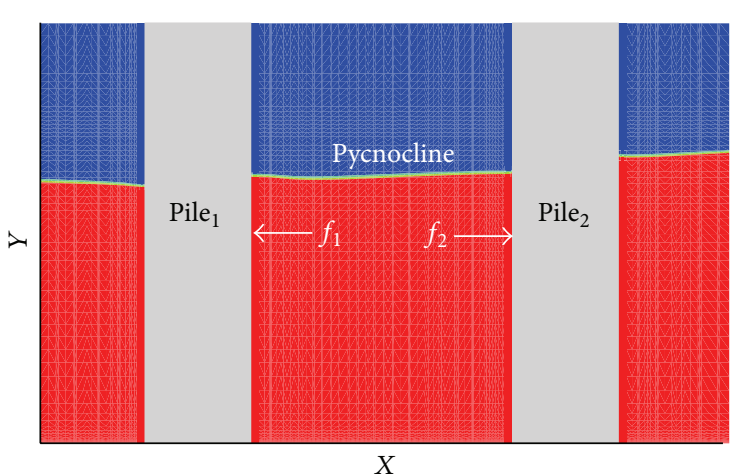

FIGURE 14: Schematic diagram showing the interaction forces between two piles.

4.3. Analysis of the Flow Field Characteristic around Pile for Various Tandem Arrangements. (a) At the gap of 1.5D: Figure 17(a) illustrates the instantaneous vertical-averaged vorticity contours with streamlines in the upper layer. It can be noticed that Pile ${ }_{2}$ is inside the wake formed behind Pile ${ }_{1}$, making the downstream body be immersed in a region of 


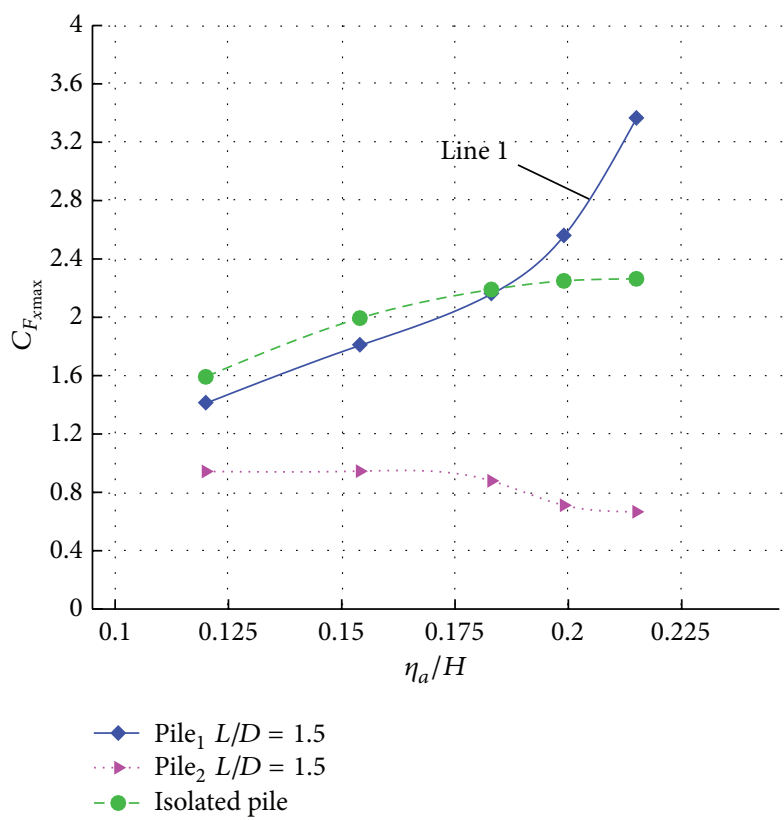

(a)

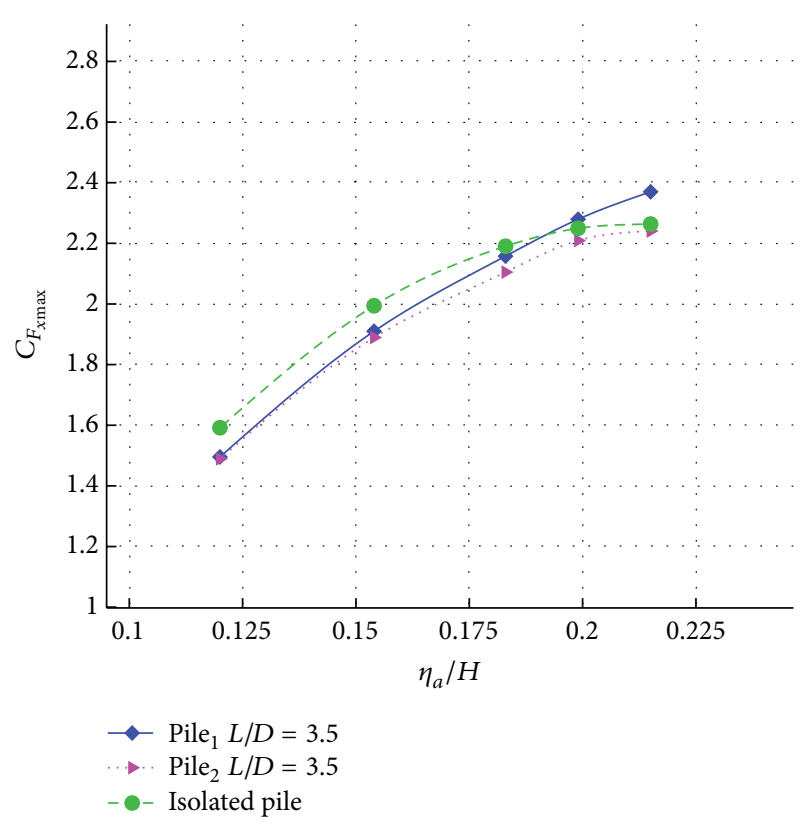

(b)

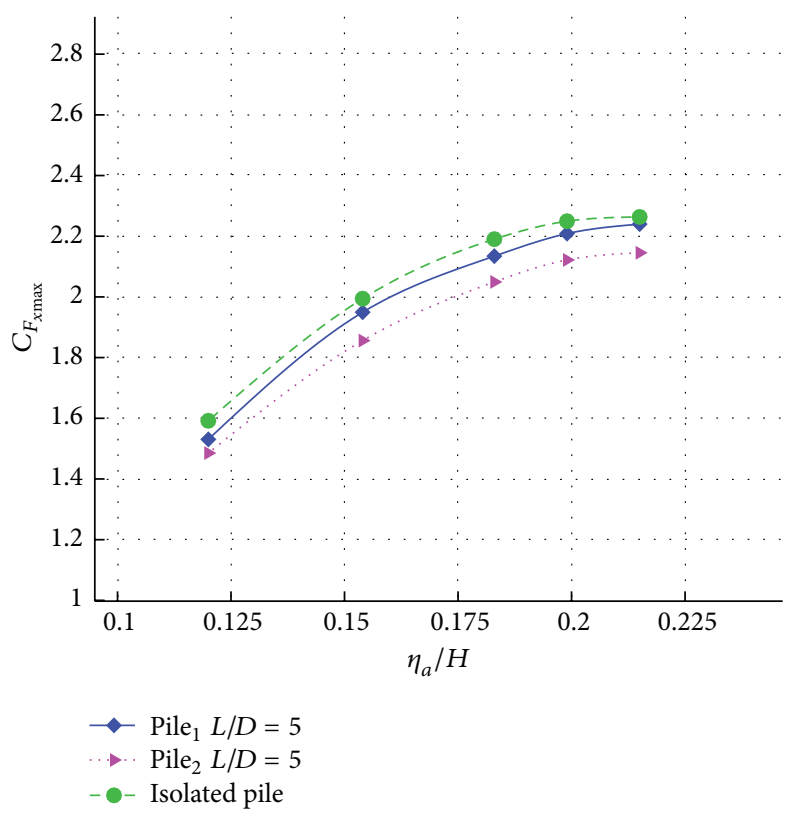

(c)

FIgURE 15: Dependence on $\eta_{a} / H$ of $C_{F_{x \max }}$ on the tandem piles compared with isolated one: (a) $L / D=1.5$, (b) $L / D=3.5$, and (c) $L / D=5$.

low pressure; in the lower layer, by comparing the contours of $L / D=1.5$ with a single pile as illustrated in Figures 18(a) and 18(b), we find that the instantaneous verticalaveraged adverse pressure gradient (Zone B) in front of Pile 2 is obviously weakened by the existence of Pile $_{1}$.

(b) At the gap of $2.5 \mathrm{D}$ : in the upper layer, it can be seen clearly in Figure 17(b) that Pile $_{2}$ moves out of the lowerpressure and recirculation area, continuing to be hit by the vortices generated behind Pile ${ }_{1}$; in the lower layer, Zone A and Zone $B$ of $L / D=2.5$ shown in Figure 18(c) are in a similar situation to that of the single pile in Figure 18(b), which reveals that the effect of pressure gradient on $\mathrm{Pile}_{2}$ can be ignored at this gap. Therefore, only the vortex effect in the upper layer should be taken into account for gaps $\geq 2.5$.

(c) At the gap of $3.5 \mathrm{D}$ : in the upper layer, the hydrodynamic interaction still exists between two piles (see Figure $17(\mathrm{c})$ ). But the hitting force exerted by vortices decreases continuously with the spacing increasing accordingly.

(d) At the gap of 5D: plot of instantaneous verticalaveraged vorticity contours in Figure 17(d) shows that the interaction between two piles tends to vanish when the spacing is large enough. 


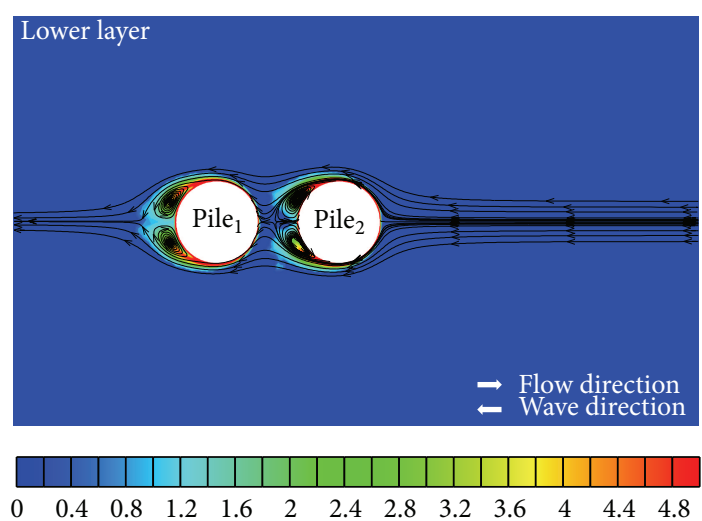

(a)

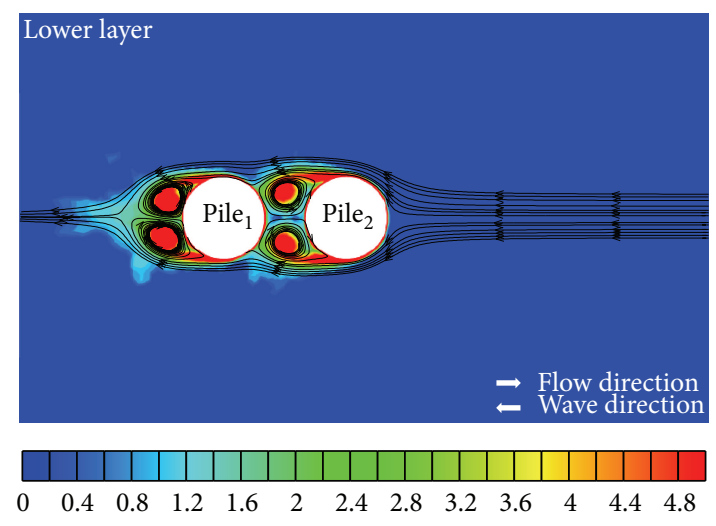

(b)

FIGURE 16: Instantaneous vertical-averaged vorticity contours with streamlines for diverse $\eta_{a} / H$ when $C_{F_{x}}$ is the maximum, $L / D=1.5:(a)$ $\eta_{a} / H=0.12$, (b) $\eta_{a} / H=0.215$, in the lower layer.

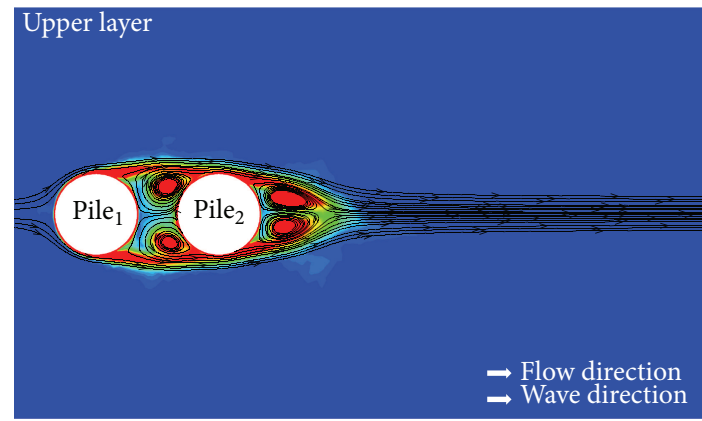

$\begin{array}{llllllllllllll}0 & 0.4 & 0.8 & 1.2 & 1.6 & 2 & 2.4 & 2.8 & 3.2 & 3.6 & 4 & 4.4 & 4.8\end{array}$

(a)

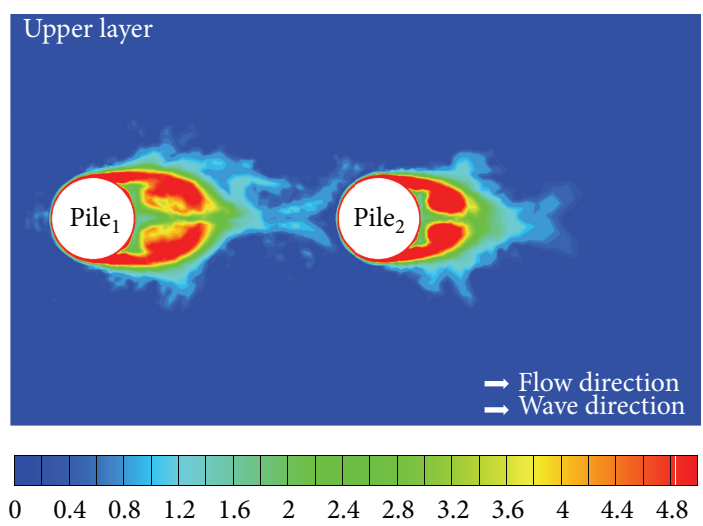

(c)
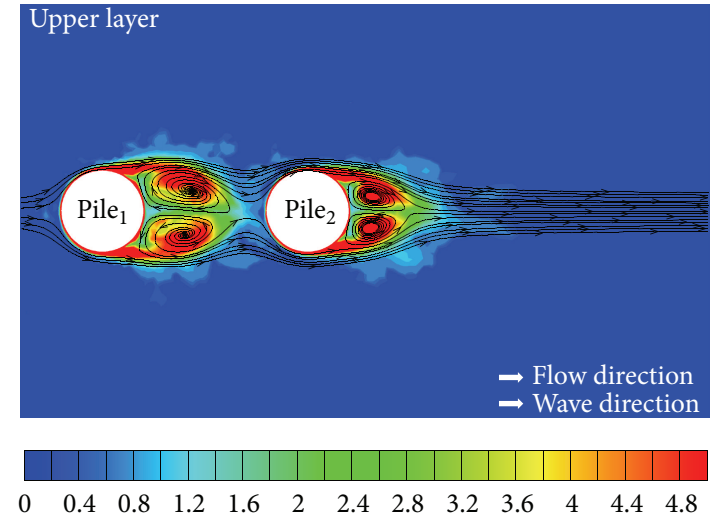

(b)

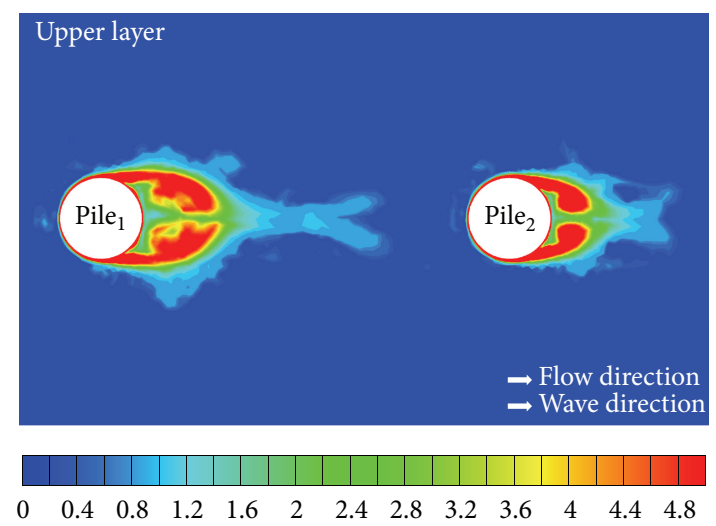

(d)

FIGURE 17: Instantaneous vertical-averaged vorticity contours and streamlines for diverse $L / D$ when $C_{F_{x}}$ is the maximum, $\eta_{a} / H=0.5:(a)$ $L / D=1.5$, (b) $L / D=2.5$, (c) $L / D=3.5$, and (d) $L / D=5$, in the upper layer.

Similarly, the spacing between two cylinders for Pile 2 can also be classified into three interference regions distinguished by different hydrodynamic interactions: $1.5 \leq L / D<2.5$, $2.5 \leq L / D<3.5$, and $3.5 \leq L / D \leq 5$. The force behaviors of $\mathrm{Pile}_{2}$ in these regions will also be further discussed below.
4.4. ISWs Forces on Pile 2 . The three-dimensional simulation results of $C_{F_{x \max }}$ on Pile 2 are shown in Figure 19. It can be seen that all the five configurations exhibit a similar behavior over $L / D$, except that the systematic differences in the values of $C_{F_{x \max }}$ are observed along the entire $L / D$ range investigated. 


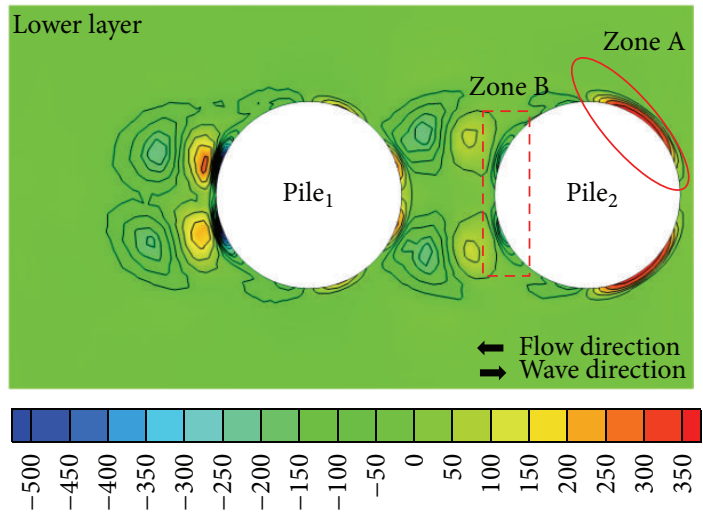

(a)

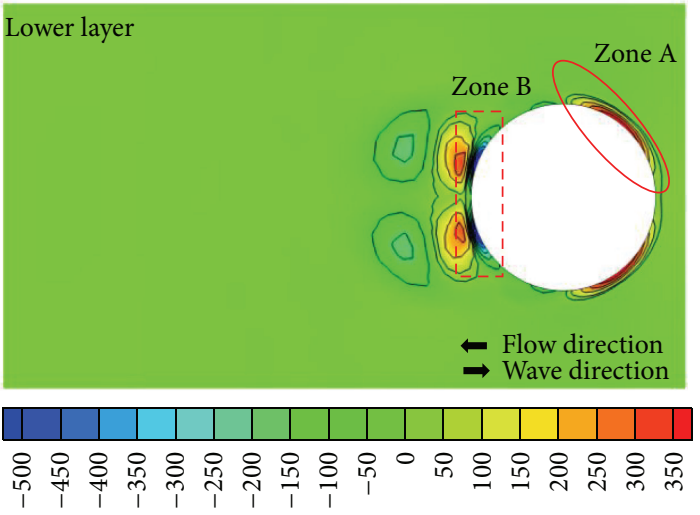

(b)

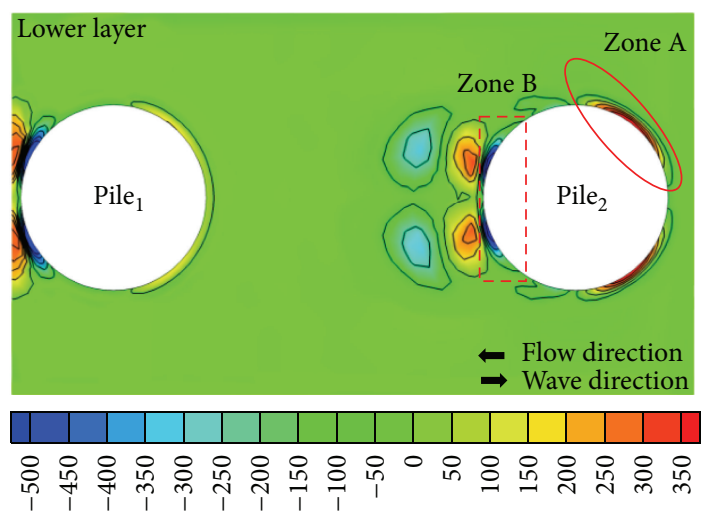

(c)

FIGURE 18: Instantaneous vertical-averaged pressure gradient contours when $C_{F_{x}}$ is the maximum, $\eta_{a}=0.215$ : (a) $L / D=1.5$, (b) isolated pile, and (c) $L / D=2.5$, in the lower layer.

By comparing the following five curves of the measured $C_{F_{x \max }}$ of Pile ${ }_{2}$, the systematic difference between two adjacent curves diminishes gradually with the increase of $\eta_{a} / H$. It indicates that the influence by wave amplitude on horizontal forces tends to depression. Similar phenomenon can also be found in Figure 13 of $C_{F_{x \max }}$ on Pile 1 .

For gaps in the range of $1.5 \leq L / D \leq 2.5$, as illustrated in Figure 19, $C_{F_{x_{\max }}}$ of each case increases rapidly with the increasing spacing. First, $C_{F_{x \max }}$ meets its minimum at the gap of $1.5 D$. This fact can be understood by the weakening of the adverse pressure gradient and the influence of lowerpressure area (studied in Section 4.3(a)), which makes $f_{2}$ (defined in Figure 14) on Pile $_{2}$ decrease in both layers at this gap. When the gap reaches $2.5 \mathrm{D}$ (see in Figure 17(b)) Pile ${ }_{2}$ 's moving out of the lower-pressure area and continuing to be hit by vortices induce rapid increase in $f_{2}$ and cause $C_{F_{x \max }}$ to increase sharply to its peak. Afterwards, the impact force by vortices continually decreases as the spacing is being enlarged to $3.5 D$. So the curves for all the configurations present a descending trend between the gap of $2.5 D$ and $3.5 D$.

If the gap is further enlarged to $5 D$, the variation of $C_{F_{x \max }}$ with cylinder centre-to-centre spacing is very small as shown

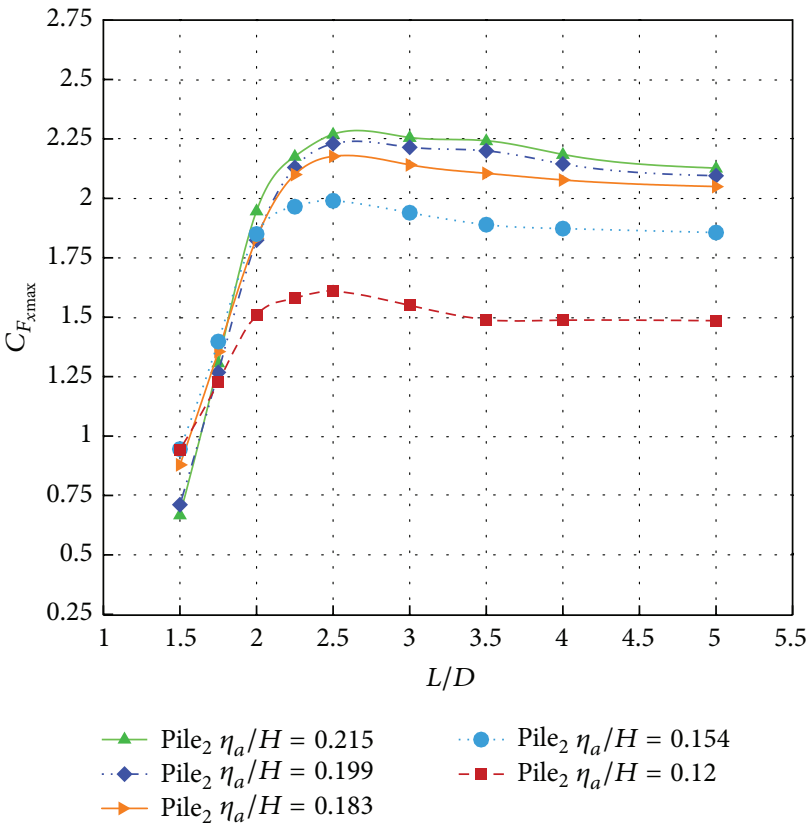

FIGURE 19: $C_{F_{x \max }}$ as a function of $L / D$ for different $\eta_{a} / H$ of Pile $_{2}$. 
in Figure 19. Plot of instantaneous vertical-averaged vorticity contours (see in Figure 14(d)) shows that interaction between two cylinders at the gap of $5 D$ gradually decreases into a noninteracting state. Therefore, it is clear that, at this gap, the horizontal force on the multiple slender structure is altered in a similar way to that observed in the isolated cylinder case.

\section{Conclusion}

A three-dimensional numerical wave flume is adopted to investigate the ISWs force exerted upon two tandem cylinders. Different centre-to-centre spacings $(L)$ between two piles along with various ISWs amplitudes $\left(\eta_{a}\right)$ are considered in current study, which can provide a better understanding of the hydrodynamic interference and the ISWs force behaviors of a multiple slender structure. The results for two piles are also compared with that of an isolated cylinder present in the same environment. From study conducted in this paper, the conclusions can be made as follows:

(1) Being different from single-layer flow environment, the horizontal currents induced by internal waves are in the opposite direction between upper and lower layers. So the contributing factors (the effects of vortices and pressure gradient) related to the loading on either pile should be studied separately in each layer at different spacings.

(2) Hydrodynamic interactions regions are sensitive to $L / D$, and the numerical results obtained from these regions are summarized below:

(a) For $1.5 \leq L / D \leq 3.5$, strong mutual interference appears between two cylinders. Within this interval, the changes in vorticity fields and pressure gradient fields are considered as the key roles in influencing the force behaviors of two piles. However, the effect of pressure gradient can be ignored when $L / D \geq 2$ for the upstream pile $\left(\right.$ Pile $\left._{1}\right)$ and $L / D \geq 2.5$ for the downstream pile $\left(\right.$ Pile $\left._{2}\right)$. The dimensionless global horizontal force amplitude $\left(C_{F_{x \max }}\right)$ on Pile 1 reaches its maximum at $1.5 \mathrm{D}$ and then touches minimum at $2 D$, while $C_{F_{x \max }}$ on Pile $_{2}$ peaks at $2.5 \mathrm{D}$ after experiencing its minimum at $1.5 D$.

(b) At the range of $3.5<L / D<5$, the two cylinders keep on influencing each other in a weakinterference state. $C_{F_{x \max }}$ on both piles illustrates slightly declining trends, because the impact force of vortices exerted upon piles decreases gradually as the gap is further enlarged to $5 D$.

(c) Beyond the gap of $5 D$, the interaction between two piles progressively decreases into a noninteracting state, and $C_{F_{x \max }}$ on the multiple slender structure is presented in a similar way to that on the isolated cylinder.

(3) An important thing to note is that the inflection points in $C_{F_{x \max }}$ trend curves vary for different piles: $L / D=2$ for Pile $_{1}$ and $L / D=2.5$ for Pile $_{2}$. This is because the near wake between two piles in the upper layer covers a wider range than that in the lower layer, evidenced by comparing Figure 12(b) with Figure 14(b). Pile 2 moves out of the low-pressure region at around the gap of $2.5 D$, while for Pile $_{1}$ at around $2 D$.

\section{Competing Interests}

The authors declare that they have no competing interests.

\section{Acknowledgments}

This work was supported by the National Natural Science Foundation of China (Grants nos. 51479058 and 51309085), the special fund for Public Welfare of Water Resources Ministry (Grant no. 201501007), the State Key Program of National Natural Science of China (Grant no. 51239003), the 111 Project (Grant no. B12032), the Fundamental Research Funds for the Central Universities (2014B36114), and the Innovation Project of the Scientific Research for College Graduates of Jiangsu Province (KYLX 0467).

\section{References}

[1] S. Petillo and H. Schmidt, "Exploiting adaptive and collaborative AUV autonomy for detection and characterization of internal waves," IEEE Journal of Oceanic Engineering, vol. 39, no. 1, pp. 150-164, 2014.

[2] N. V. Kurup, S. Shi, Z. Shi, W. Miao, and L. Jiang, "Study of nonlinear internal waves and impact on offshore drilling units," in Proceedings of the ASME 2011 30th International Conference on Ocean, Offshore and Arctic Engineering, vol. 1, pp. 831-840, Rotterdam, Netherlands, June 2011.

[3] E. V. Ermanyuk and N. V. Gavrilov, "Experimental study of the dynamic effect of an internal solitary wave on a submerged circular cylinder," Journal of Applied Mechanics and Technical Physics, vol. 46, no. 6, pp. 800-806, 2005.

[4] G. Wei, H. Du, X. H. Xu et al., "Experimental investigation of the generation of large-amplitude internal solitary wave and its interaction with a submerged slender body," Science China Physics, Mechanics and Astronomy, vol. 57, no. 2, pp. 301-310, 2014.

[5] J. Xie, J. C. Xu, and S. Q. Cai, "A numerical study of the load on cylindrical piles exerted by internal solitary waves," Journal of Fluids and Structures, vol. 27, no. 8, pp. 1252-1261, 2011.

[6] S. Q. Cai, J. C. Xu, Z. Chen, J. Xie, X. Deng, and H. Lv, “The effect of a seasonal stratification variation on the load exerted by internal solitary waves on a cylindrical pile," Acta Oceanologica Sinica, vol. 33, no. 7, pp. 21-26, 2014.

[7] S. Cai, X. Long, and Z. Gan, "A method to estimate the forces exerted by internal solitons on cylindrical piles," Ocean Engineering, vol. 30, no. 5, pp. 673-689, 2003.

[8] S. Q. Cai, S. G. Wang, and X. M. Long, "A simple estimation of the force exerted by internal solitons on cylindrical piles," Ocean Engineering, vol. 33, no. 7, pp. 974-980, 2006.

[9] Z. Xu, B. Yin, H. Yang, and J. Qi, "Depression and elevation internal solitary waves in a two-layer fluid and their forces on cylindrical piles," Chinese Journal of Oceanology and Limnology, vol. 30, no. 4, pp. 703-712, 2012. 
[10] Z. J. Si, Y. L. Zhang, and Z. S. Fan, "A numerical simulation of shear forces and torques exerted by large-amplitude internal solitary waves on a rigid pile in South China Sea," Applied Ocean Research, vol. 37, pp. 127-132, 2012.

[11] C. M. Linton and M. McIver, "The interaction of waves with horizontal cylinders in two-layer fluids," Journal of Fluid Mechanics, vol. 304, pp. 213-229, 1995.

[12] J. R. Cadby and C. M. Linton, "Three-dimensional water-wave scattering in two-layer fluids," Journal of Fluid Mechanics, vol. 423, pp. 155-173, 2000.

[13] I. V. Sturova, "Radiation loads on interface piercing cylinder in a two-layer fluid of finite depth by a coupled element technique," in Proceedings of the 8th International Conference on Numerical Ship Hydrodynamics, vol. 2, pp. 80-91, Busan, Republic of Korea, 2003.

[14] T. Du, L. Sun, Y. Zhang, X. Bao, and X. Fang, "An estimation of internal soliton forces on a pile in the ocean," Journal of Ocean University of China, vol. 6, no. 2, pp. 101-106, 2007.

[15] Z. J. Song, B. Teng, Y. Gou et al., "Comparisons of internal solitary wave and surface wave actions on marine structures and their responses," Applied Ocean Research, vol. 33, no. 2, pp. 120129, 2011.

[16] H. Q. Zhang and J. C. Li, Wave Loading on Floating Platforms by Internal Solitary Waves, Springer, Berlin, Germany, 2007.

[17] L. Bonakdar, H. Oumeraci, and A. Etemad-Shahidi, "Wave load formulae for prediction of wave-induced forces on a slender pile within pile groups," Coastal Engineering, vol. 102, pp. 4968, 2015.

[18] M. M. Zdravkovich, "Review of flow interference between two circular cylinders in various arrangements," Journal of Fluids Engineering, vol. 99, no. 4, pp. 618-633, 1977.

[19] M. Mahbub Alam and Y. Zhou, "Strouhal numbers, forces and flow structures around two tandem cylinders of different diameters," Journal of Fluids and Structures, vol. 24, no. 4, pp. 505-526, 2008.

[20] J. R. Meneghini, F. Saltara, C. L. R. Siqueira, and J. A. Ferrari Jr., "Numerical simulation of flow interference between two circular cylinders in tandem and side-by-side arrangements," Journal of Fluids \& Structures, vol. 15, no. 2, pp. 327-350, 2001.

[21] H. Gopalan and R. Jaiman, "Numerical study of the flow interference between tandem cylinders employing non-linear hybrid URANS-LES methods," Journal of Wind Engineering \& Industrial Aerodynamics, vol. 142, pp. 111-129, 2015.

[22] M. Germano, U. Piomelli, P. Moin, and W. H. Cabot, "A dynamic subgrid-scale eddy viscosity model," Physics of Fluids A, vol. 3, no. 7, pp. 1760-1765, 1991.

[23] H. Zhu, L. Wang, and H. Tang, "Large-eddy simulation of the generation and propagation of internal solitary waves," Science China Physics, Mechanics \& Astronomy, vol. 57, no. 6, pp. 11281136, 2014.

[24] C.-Y. Chen, J. R.-C. Hsu, C.-W. Chen, H.-H. Chen, C.-F. Kuo, and M.-H. Cheng, "Generation of internal solitary wave by gravity collapse," Journal of Marine Science and Technology, vol. 15, no. 1, pp. 1-7, 2007.

[25] Z.-H. Lin and J.-B. Song, "Numerical studies of internal solitary wave generation and evolution by gravity collapse," Journal of Hydrodynamics, vol. 24, no. 4, pp. 541-553, 2012.

[26] A. F. Shchepetkin, "An adaptive, Courant-number-dependent implicit scheme for vertical advection in oceanic modeling," Ocean Modelling, vol. 91, pp. 38-69, 2015.
[27] J. R. Morison, J. W. Johnson, and S. A. Schaaf, "The force exerted by surface waves on piles," Journal of Petroleum Technology, vol. 2, no. 5, pp. 149-154, 2013.

[28] G. H. Keulegan and L. H. Carpenter, "Forces on cylinders and plates in an oscillating fluid," Journal of Research of the National Bureau of Standards, vol. 60, no. 5, pp. 423-440, 1958.

[29] T. Sarpkaya and I. Michael, "Mechanics of wave forces on offshore structures," International Journal of Soil Dynamics and Earthquake Engineering, vol. 60, no. 5, pp. 215-218, 1981.

[30] D. Canuto and K. Taira, "Two-dimensional compressible viscous flow around a circular cylinder," Journal of Fluid Mechanics, vol. 785, pp. 349-371, 2015.

[31] M. M. Zdravkovich, "The effects of interference between circular cylinders in cross flow," Journal of Fluids and Structures, vol. 1, no. 2, pp. 239-261, 1987.

[32] Y. N. Fu, X. Z. Zhao, X. G. Wang, and F. Cao, "Computation of flow past an in-line oscillating circular cylinder and a stationary cylinder in tandem using a CIP-based model," Mathematical Problems in Engineering, vol. 2015, Article ID 568176, 9 pages, 2015.

[33] T. Igarashi, "Characteristics of the flow around two circular cylinders arranged in tandem: 1st report," Bulletin of JSME, vol. 24, no. 188 , pp. 323-331, 1981.

[34] T. Tsutsui, T. Igarashi, and K. Kamemoto, "Interactive flow around two circular cylinders of different diameters at close proximity. Experiment and numerical analysis by vortex method," Journal of Wind Engineering and Industrial Aerodynamics, vol. 69-71, no. 594, pp. 279-291, 1997.

[35] B. S. Carmo and J. R. Meneghini, "Numerical investigation of the flow around two circular cylinders in tandem," Journal of Fluids and Structures, vol. 22, no. 6-7, pp. 979-988, 2006. 


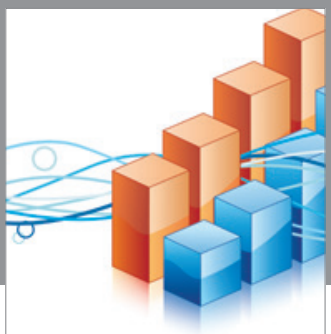

Advances in

Operations Research

vatem alat4

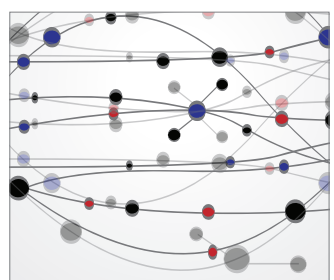

\section{The Scientific} World Journal
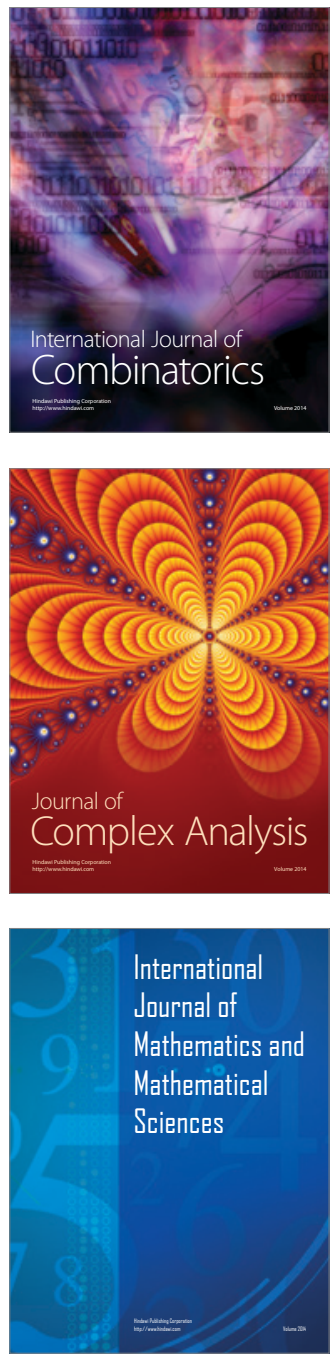
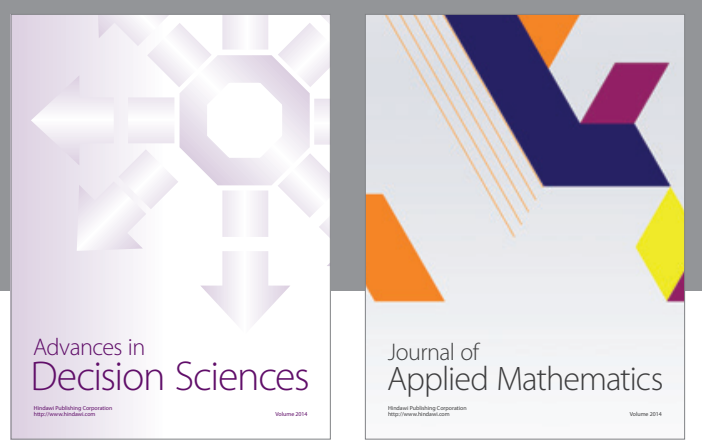

Algebra

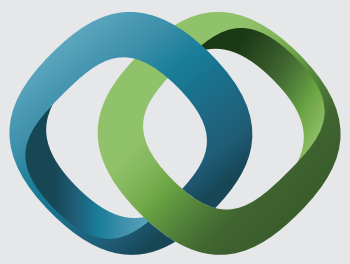

\section{Hindawi}

Submit your manuscripts at

http://www.hindawi.com
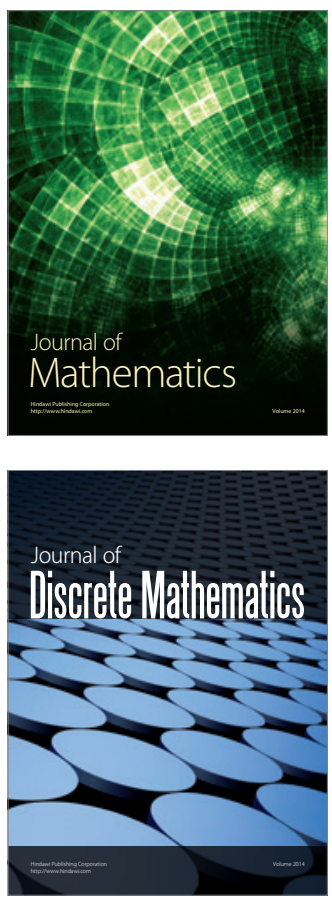

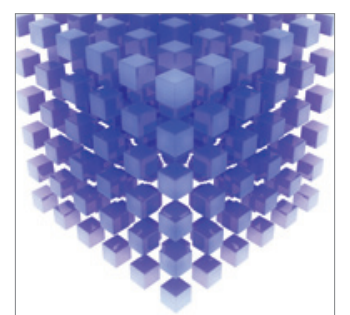

Mathematical Problems in Engineering
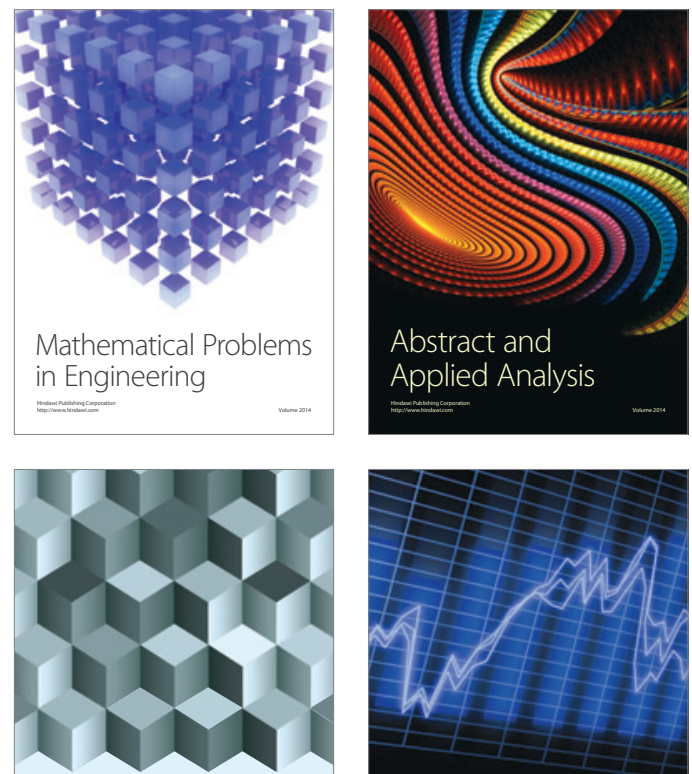

Journal of

Function Spaces

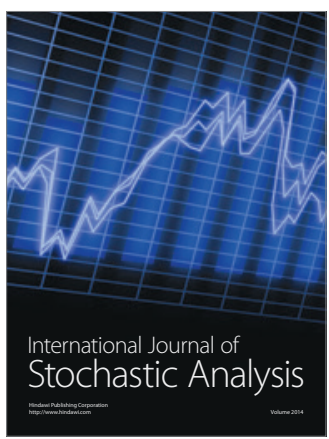

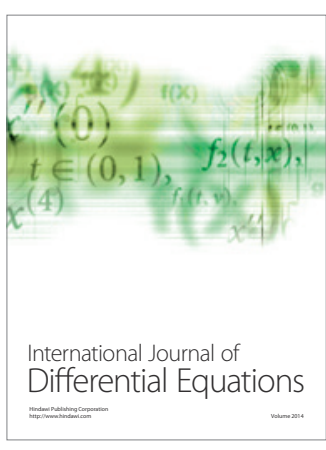
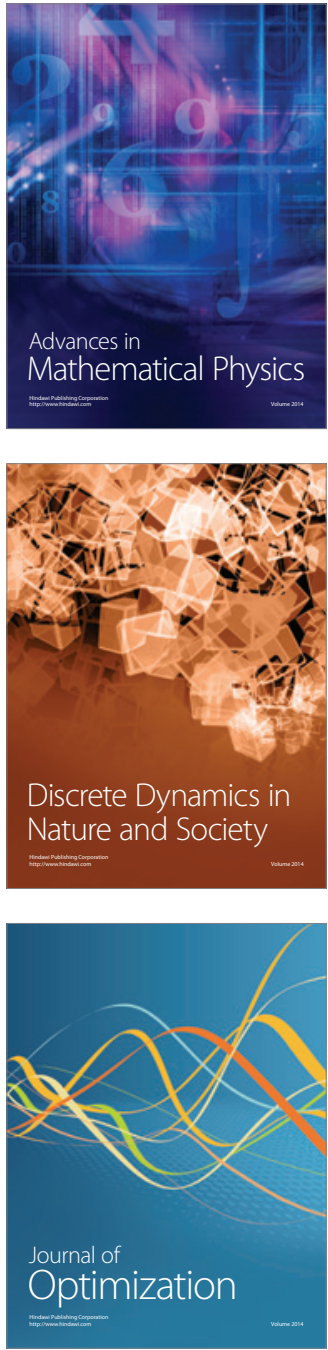